\title{
Polarization-dependent Strain and Twist sensors based on tilted fiber Bragg grating
}

\author{
By
}

\begin{abstract}
A thesis submitted to
The Faculty of Graduate Studies and Research in partial fulfilment of the degree requirements of Master of Applied Science
\end{abstract}

Ottawa-Carleton Institute for Electrical and Computer Engineering

\author{
Department of Electronics \\ Carleton University \\ Ottawa, Ontario, Canada \\ January 2014 \\ Copyright (C) \\ 2014 - Ruichao Li
}




\section{Abstract}

Tilted fiber Bragg grating (TFBG) shows various advantages in sensing parameters such as, strain, temperature and twist angle. In particular, multiple data collected from cladding modes of TFBG provide a multi-functional modality. According to this feature, a temperature-independent strain sensor and a high sensitivity twist sensor based on TFBG are introduced in this thesis. Both strain and twist sensors are designed with simplex structures by employing sole TFBG inscribed inside standard single mode fiber respectively.

The relative wavelength shifts of $\mathrm{P}$ and $\mathrm{S}$ polarized spectra are utilized in analyzing strain sensors based on their strong temperature-independent characteristics. Linear experimental results which relate to the wavelength separation from the Bragg mode resonance are presented. Meanwhile, sensitive amplitude variations with respect to the strain applied to TFBG are also evident.

Moreover, TFBG shows high sensitivity to twist angle due to its unique structure. The sensitivity of Polarization-dependent loss (PDL) presents an obvious contrast to that of insertion loss; the comparisons are described experimentally. In particular, PDL spectrum of TFBG has much higher twist sensitivity than that of insertion loss spectrum. 


\section{Acknowledgements}

I would like to thank my supervisor, Professor Jacques Albert, who gave me the opportunity to carry out my graduate studies and accomplish this thesis. He provided the initial inspiration and critical proposals that guided me through my work. His professional assistance and patience encouraged and supported me through my research and studies.

I would also like to express my gratitude to those who helped me finish my work. In particular, I would like to thank Wenjun Zhou and Lingyun Xiong for their helpful advice. I would like to thank Yang Zhang, Albane Laronche and Lépinay Sandrine for helping me learn the correct equipment usage methods. Thanks should also go to the members of my research group, Alexander Andreyuk, Katherine Bock, Milad Abou Dakka, and Yan Zhou for creating an excellent working environment.

Finally, I would like to thank my family for their support all through my life. 


\section{Table of Contents}

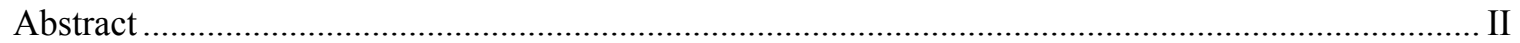

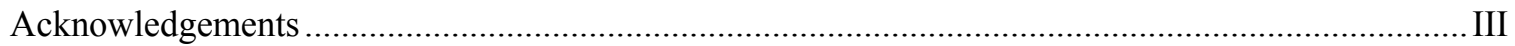

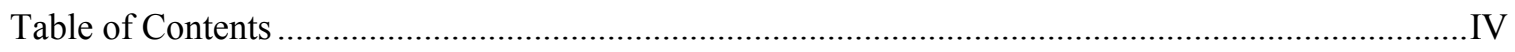

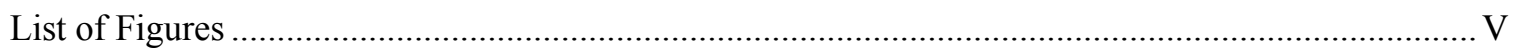

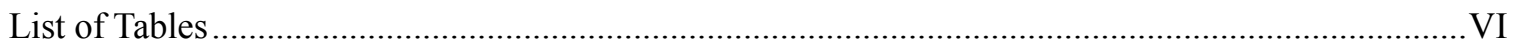

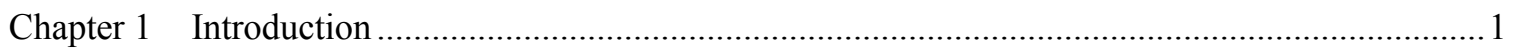

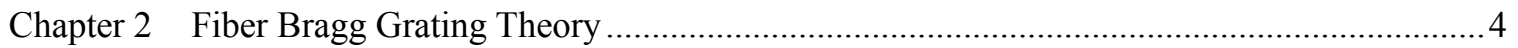

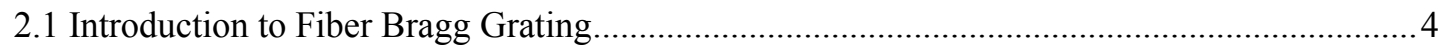

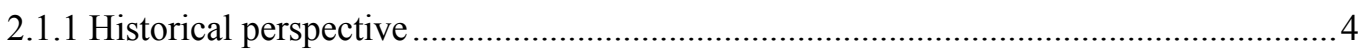

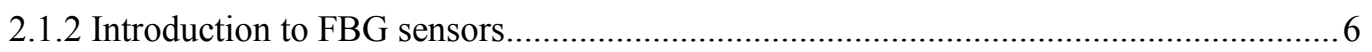

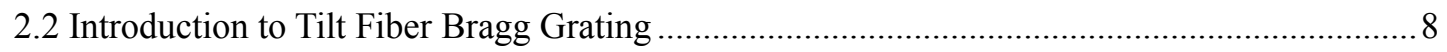

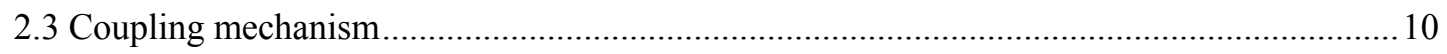

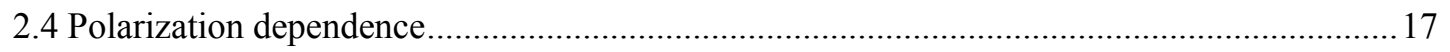

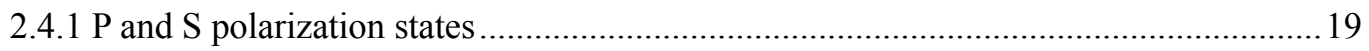

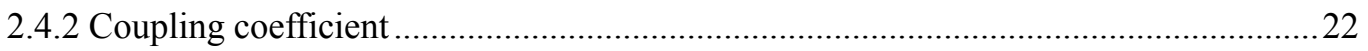

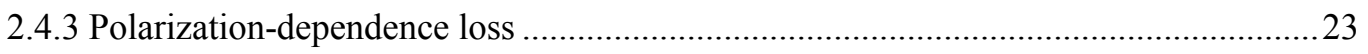

Chapter 3 Polarized Temperature-independent Strain Sensor.........................................................25

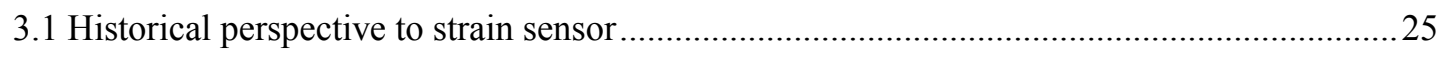

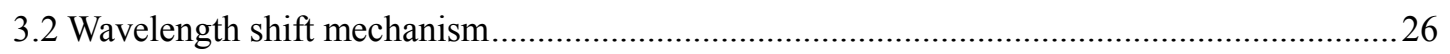

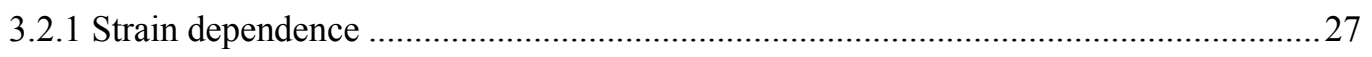

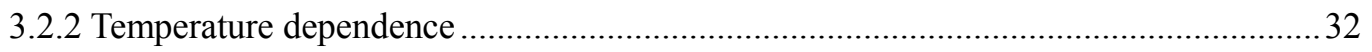

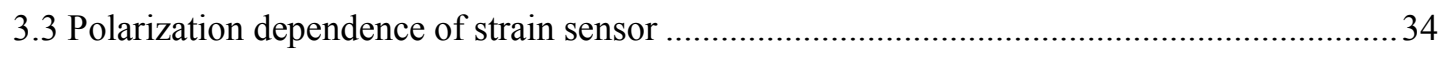

3.4 Temperature-independent strain sensor utilizing relative polarized spectrum .......................... 38

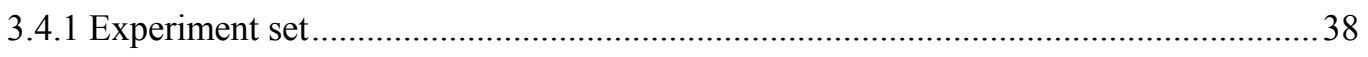

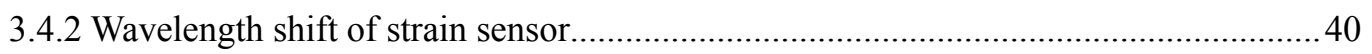

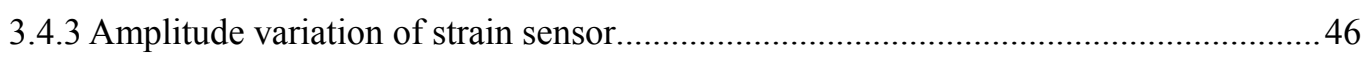

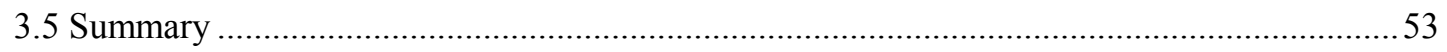

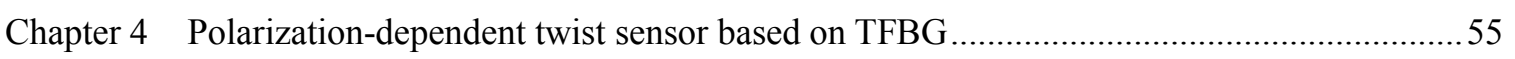

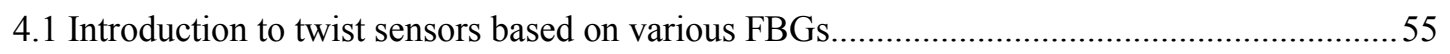

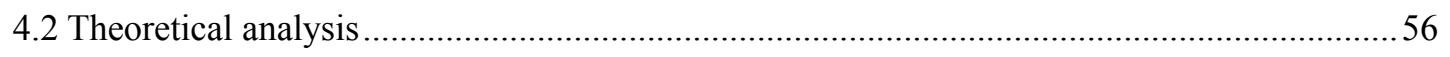

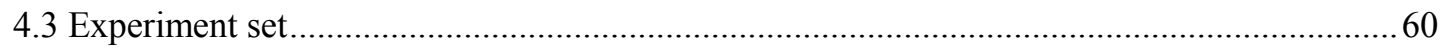

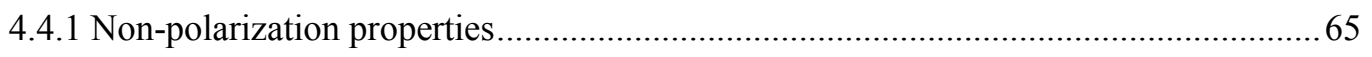

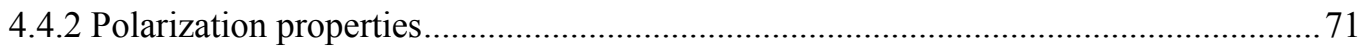

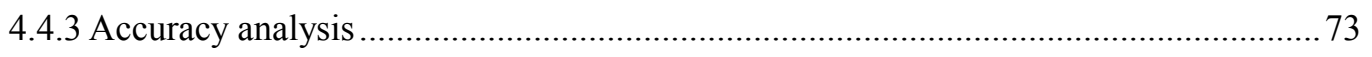

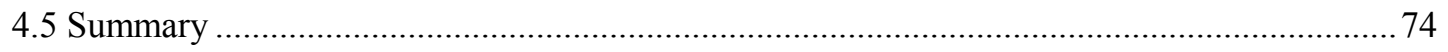

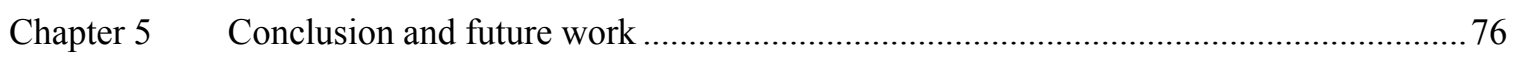

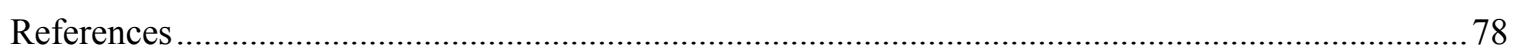




\section{List of Figures}

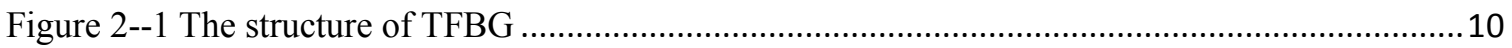

Figure 2--2 Experimentally measured Transmission spectrum of (a) FBG and (b) TFBG ...................12

Figure 2--3 The split of high order cladding modes....................................................................17

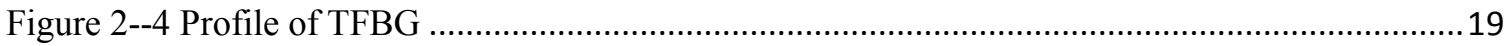

Figure 2--5 Experimentally measured Transmission spectra of $\mathrm{P}$ and $\mathrm{S}$ polarization states ................21

Figure 2--6 Experimentally measured PDL spectrum ...................................................................23

Figure 3--1 Experimentally measured Wavelength shift caused by strain ........................................ 31

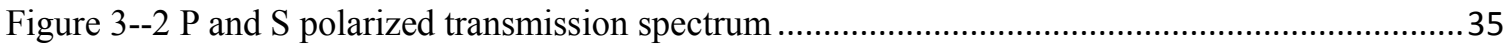

Figure 3--3 Relative spectra of $\mathrm{P}$ and $\mathrm{S}$ polarization states near the ghost mode while the applied

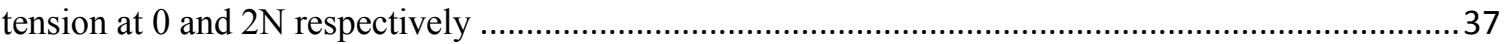

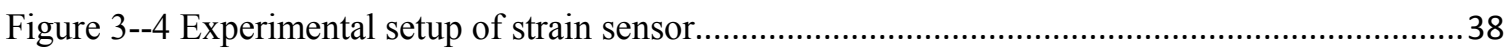

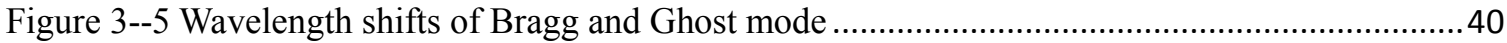

Figure 3--6 Relative wavelength shifts of $\mathrm{P}$ and $\mathrm{S}$ polarization states .............................................. 41

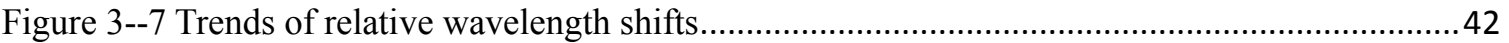

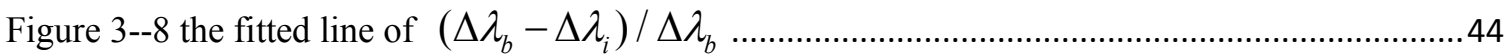

Figure 3--9 The ratio $\left(\Delta \lambda_{b}-\Delta \lambda_{i}\right) / \Delta \lambda_{b}$ at different wavelength...............................................46

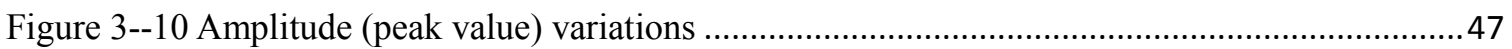

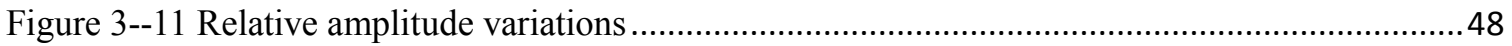

Figure 3--12 The overlapped $\mathrm{P}$ and S polarized spectra at different wavelengths ...............................51

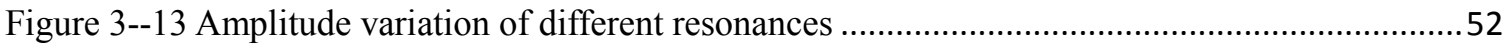

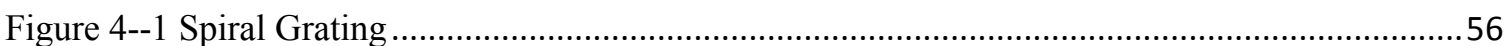

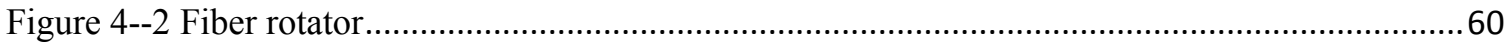

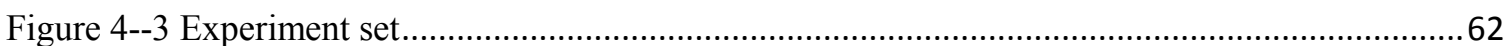

Figure 4--4 Transmission spectra versus torsion angle applied to the connected fiber ........................64

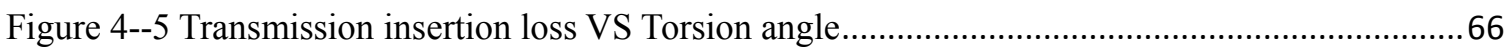

Figure 4--6 Wavelength shifts and Relative amplitude variations......................................................6

Figure 4--7 The deformations of resonances caused by torsion angle ............................................... 70

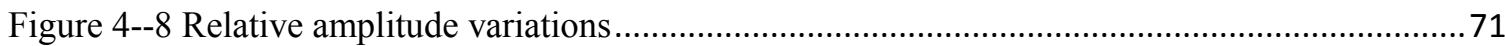

Figure 4--9 Average amplitude variations of all cladding modes between $\mathrm{CCW}$ and $\mathrm{CW}$...................72 


\section{List of Tables}

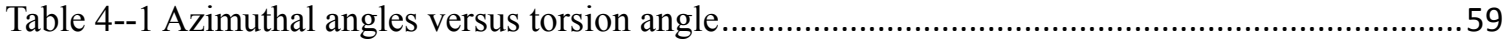

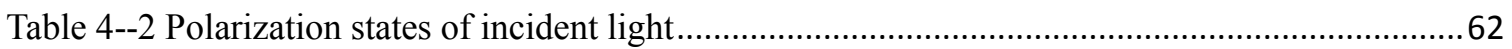

Table 4--3 The variations of Bragg and ghost mode resonances............................................................66 


\section{Chapter 1 Introduction}

Sensors are very important components in modern industry. Nowadays, sensors have become more sensitive, precise, compact, and intelligent. Especially, since the possibility of optical fiber sensors being embedded into reinforced concrete buildings and other structures was demonstrated by Mendez et al in 1989 [1], the new kinds of sensors which employ FBG as the sensitive element have been widely applied in the USA, Canada and Europe since the 1990s. [2] [3]

Sensors based on Fiber Bragg Grating (FBG), as a new member of the sensor family, have attracted widespread attention as a result of various advantages, including their perfect interference immunity to electric and magnetic fields; excellent mechanical behaviours such as small size, flexible shape and lightweight; and outstanding chemical properties consisting of good resistance to water, corrosion and high temperature. In particular, the sensor element based on FBG can be easily embedded into composite material or structures so that it can conveniently sense the properties of internal structures of buildings, pipelines, tunnels etc., to measure strain and temperature, among other things. In addition to the advantages of the sensitive element based on FBG, the sensor system consists of a control center which involves interrogation devices, a transmission light path based on optical fibers and sensitive elements (arrays) which provides the rapid 
and accurate information of the monitored target parameters. Consequently, the information carried by light is in real time (the parameters of the monitored target are displayed in seconds as a result of the very short delay during propagation and demodulation) and the light propagated inside the optical fiber is secluded (so that the disturbance by the external environment around transmission light path based on optical fiber is nonexistent).

A FBG sensor works as a result of the response from the spectral characteristics of FBG (amplitude, wavelength and polarization of transmission or reflection spectra) which relates to the effect of the monitored parameters. Actually, multiform variants of FBG, such as chirped fiber grating [4], long-period FBG (LPG) [5] [6] , tilt FBG (TFBG) [7] and some special in-fiber gratings [8] [9] show different spectral characteristics. There is a large amount of literature that analyzes the sensing properties of different FBGs from which TFBG shows unique spectral characteristics which strongly relate to the polarization states, due to the introduction of the tilted angle which breaks the fiber cylindrical symmetry of grating planes. In particular, as a result of the special quasi-three-dimensional structure of TFBG, complex strong couplings are enhanced and become obvious so that they are easily monitored as cladding mode resonances in the transmission spectrum of TFBG. Compared to the transmission or reflection spectra of normal FBG, which only displace the Bragg mode resonance, the numerous cladding mode resonances of TFBG are strong, considerable and observable and offer a 
multi-functional model allowing various functions to be implemented by analyzing complex data collected from coupling resonances [10] [11].

The objective of this thesis is to simply describe new strain and twist sensors based on TFBG by analyzing the complex data collected from numerous cladding mode resonances. Chapter 2 will introduce the history, properties, and advantages of FBG and TFBG sensors. In particular, the coupling and polarization mechanism of TFBG will be presented in order to theoretical analyze the characteristics of TFBG sensors. Chapter 3 will introduce a new kind of temperature-independent strain sensor which employs the polarized transmission spectra of TFBG in order to reduce the disturbances caused by various reasons. A twist sensor based on TFBG will be introduced in Chapter 4. This sensor employs the polarization-dependence loss (PDL) spectrum to monitor the response to torsion angle as a result of the strong polarization-dependence of twist sensors based on TFBG. Lastly, the conclusion will be presented in Chapter 5. A problem to be solved will be presented for future work. 


\section{Chapter 2 Fiber Bragg Grating Theory}

\subsection{Introduction to Fiber Bragg Grating}

Fiber Bragg Grating (FBG) is a periodic modulation of the effective index which distributes along a segment of fiber core. It can be seen as a selective mirror of wavelength which reflects the particular wavelength of incident light and transmits others. In other words, a FBG is an in-fiber grating which acts like a bandwidth filter or reflector. Based on this feature, a FBG can be utilized in various fields as a unique optical passive component [12] [13] [14] [15] [16]; it can be used as a wavelength division multiplexing filter or a dispersion compensator in telecommunications; a wavelength-selective filter which selects required wavelength of light and removes others in fiber amplifiers; or a narrow-band and high-reflectance mirror in optical sensor applications. In general, FBG plays an important role in modern optical applications.

\subsubsection{Historical perspective}

In the 1970s, the attenuation of optical fiber had been reduced from $1000 \mathrm{~dB} /$ kilometer to $20 \mathrm{~dB} /$ kilometer, according to the contribution from Charles K. Kao, who is known as the father of Fiber Optic Communications [17]. Since that time, optical fiber communication technology began transmitting information over long distances. At the same time, it was found that when light was transmitted in optical fibers, the physical 
properties of that optical fiber changed, based on external factors, so people tried to use these effects to obtain new optical fiber applications. FBG was one of the most important inventions at that time. The first FBG was demonstrated by K.O. Hill and his co-workers at the Communications Research Centre, Ottawa, Canada in 1978 [18]. They observed and reported the variation of reflection index while they injected a $488 \mathrm{~nm}$ argon ion laser into a germanosilicate fiber. They found that the transmission light intensity of the germanosilicate fiber decreased sharply along with the increase in exposure time while the laser was emitted into the fiber. At the same time, the reflection light intensity increased. In other words, a grating formed inside the core of the fiber through this exposure.

However, the new technology of FBG remained dormant for a decade after its discovery because of the limitation of the writing technique. This limitation was broken in 1989 by the discovery of UV side-written FBG method reported by Gerald Meltz and his colleagues from the United Technologies Research Center [19]. The new FBG side-written technique not only effectively improved the efficiency of FBG's written process, but also ensured the period of FBG became controllable by adjusting the angle between the two interfering light beams while a FBG was fabricated. Due to the advance in the FBG written technique, people fabricated FBGs where the grating period situates around $1300 \mathrm{~nm}$ or $1500 \mathrm{~nm}$, which is the most commonly used wavelength in telecommunications. The new FBG side-written technique pushed the FBG to become a 
practical optical passive component and attracted interests of researchers in the field of fiber optics worldwide.

In 1993, K.O. Hill and his co-workers proposed a new FBG written technique by utilizing a phase mask grating made of silica glass [20]. They irradiated the phase mask grating using a UV laser. The diffracted light of the grating formed a periodic pattern which wrote a FBG inside the core of a photosensitive fiber placed behind the grating. The main advantage of this method was that the period of the fabricated FBG was only determined by the period of the phase mask grating, so it was easy to reduce the need of the spatial coherence of the laser sources. This method of fabricating FBG is flexible, simple to use, and economical, thus it is the primary method of FBG fabrication today.

\subsubsection{Introduction to FBG sensors}

As one of the most important passive components in the field of fiber optics, FBG plays an important role in sensor applications based on the fact that when a light is transmitted through a FBG, the properties of the transmission and reflection light (amplitude, wavelength, polarization states of coupling resonance) will change due to the external factors acting on the FBG. Therefore, various sensors based on FBG are used in monitoring various parameters, such as temperature, strain, pressure and bend. Compared to traditional sensors, sensors based on FBG have significant advantages, which are listed below: 
1) Small size: FBG is inscribed inside the core of a segment of fiber, which is normally 1-2 centimeters long, and the diameter of FBG is only about $0.15 \mathrm{~mm}$. FBG sensors can be fabricated in small size, light weight and flexible shapes. Thus, FBG sensors are suitable for environments that require small size, for example sensors used internal human medicine.

2) Easily being embedded in material and structures: FBG sensors can be easily used to measure multi-points by using the FBG sensor arrays, for the detection of internal strain, temperature, pressure, damage, etc. without affecting the structure and its properties.

3) Immune to harsh environments: FBG sensors are made of silica, which is a corrosion-resistant and insulating material. Furthermore, FBG sensors are optical passive components, which work without any electronic components. Accordingly, FBG sensors can be used in various environments such as extreme low/high temperatures, high radiation and/or where intensive corrosion is possible.

4) Real-time: the FBG sensor is an optical passive component which transmits or reflects incident light without any time delay (while the speed of light is ignored); any small fluctuation passed to the sensor causes the reaction at the monitoring terminal immediately.

5) Long distance monitoring: the insertion loss of optical fibers is very low. The maximum attenuation of standard single mode optical fiber (SMF-28) is 
$0.35 \mathrm{~dB} / \mathrm{km}$ and $0.22 \mathrm{~dB} / \mathrm{km}$ at $1310 \mathrm{~nm}$ and $1550 \mathrm{~nm}$ respectively. Furthermore, since most FBG sensors work by tracking the wavelength of transmission or reflection light, which is not subject to the effects of light attenuation, the remote signal transmits along a longer fiber eliminating the chance of errors during transmission. Due to this advantage, the FBG sensor may be mounted at a distance of several kilometers away from the position of the light source or the monitoring terminal. This application is particularly useful for monitoring pipes, long-distance tunnels and remote building structures.

Since the mid-1990s, FBG sensors have been utilized in a vast number of fields to great advantage, such as internal human medicine, or imbedded in building structures in various challenging environments. [3]

\subsection{Introduction to Tilt Fiber Bragg Grating}

Tilt Fiber Bragg Grating (TFBG) is a variant of the normal FBG where the grating planes tilt relative to the perpendicular of the fiber axis [10]. The angle between the grating planes and the perpendicular of the fiber axis, which is so called the tilted angle, give in-fiber gratings special characteristics and provide a multi-functional model. The characteristics of this special structure were discovered as early as those of FBG. However, since the tilted angle complicates the spatial structure and increases the difficulty of fabrication of in-fiber gratings, TFBG was unnoticed for over a decade, until 
the concept of TFBG was first presented by Meltz et al at the Optical Fiber Communication (OFC) conference in 1990 [21]. In particular, they found numerous couplings in FBG were enhanced while the tilted angle was introduced into the grating (consider the angle between the grating planes and the perpendicular of the fiber axis was tilted from $0^{\circ}$ (for normal $\mathrm{FBG}$ ) to an angle between $0^{\circ}$ and $90^{\circ}$ (for TFBG)). Their discovery was significant in particular because compared to normal FBG, the introduction of the tilted angle of TFBG not only altered the coupling modal from original two-dimensional structure to a quasi-three-dimensional structure, but also promised the obvious feature of multi-interactions between photons (the light coupled from each grating panels was no longer a fiber cylindrical symmetry structure inside the core of fiber) [11]. The new characteristics of TFBG aroused widespread concern from the time it was discovered by Meltz et al. However, because a high photosensitivity fiber and the high-precision were required in the fabrication of TFBG, early studies only focused on the theoretical analysis [7] [22] until 2000, when various applications based on TFBG were studied and reported. Then TFBG became an important path in the study of in-fiber grating sensors. 


\subsection{Coupling mechanism}

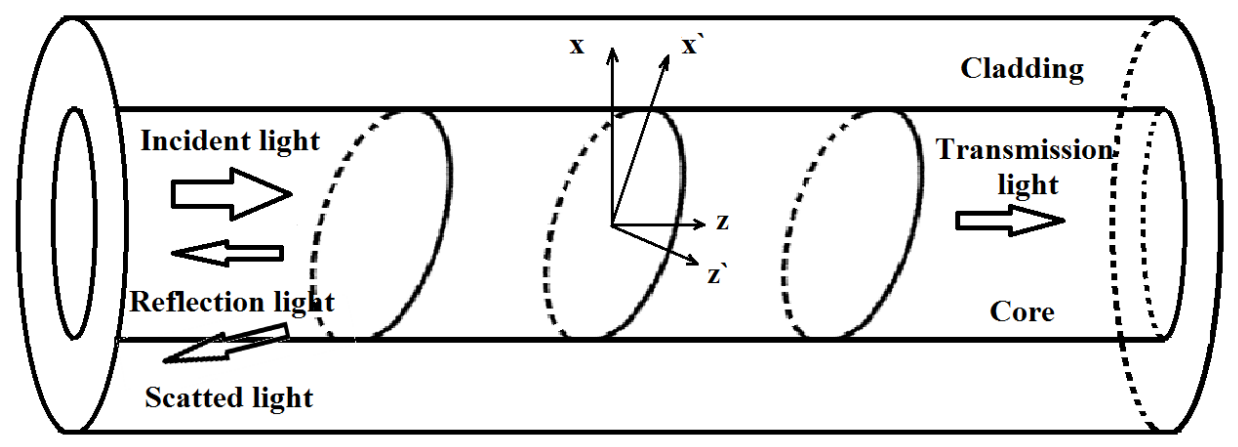

Figure 2--1 The structure of TFBG

Figure 2--1 shows the structure of a TFBG. $\theta$ is the tilted angle between the grating planes $X^{\prime}$ and the perpendicular of the fiber axis $X$ (or the wave vector of the grating $Z$ ' and the fiber axis Z). $\Lambda_{g}$ is the spatial period of the grating and $\Lambda$ is the period of the grating measured along the fiber axis. Similar to normal FBG, TFBG possesses the structure of a periodic modulation of the effective index distributes along the fiber axis. So the coupling between the forward-propagating core mode and the counter-propagating core mode, so called the Bragg mode, exists both in FBG and TFBG. However, unlike normal FBG, the tilted angle of TFBG brings great enhancement of the complex couplings, including the couplings between the forward-propagating core mode and the counter-propagating cladding modes, so called the cladding modes, and the couplings between the forward-propagating core mode and the scattered cladding modes while the diameter of the cladding of fiber is assumed to be infinite (for example, while the grating is immersed in a solution where the refraction index is higher than the 
refraction index of cladding), so called the radiation modes. The two new types of couplings show similar characteristics in several applications, such as strain, temperature and twist sensors, so in studies they are collectively referred to as cladding modes. Actually, normal FBG is seen as a single mode (Bragg mode) reflector or filter. However, compared to normal FBG, which provide simplex data from the parameters of its Bragg mode, numerous enhanced cladding modes in TFBG provide complex data from the Bragg mode and every single cladding mode. A limitation of the sensor, based on normal FBG, is that the Bragg mode, so called the core mode, only shows the parameters which act on the grating inside fiber core, such as the change of effective index or the grating period inside core. But in TFBG, the incident light beam is scattered along a particular direction into cladding or even scattered outside the fiber, so the properties of the 'scattered' light relate to polarization and external environment directly. Based on the properties, new applications based on TFBG are provided, such as the Surface Plasmon Resonance (SPR) sensor based on TFBG [23] by thin gold coating the grating. Obviously, this new application is hard to be achieved by normal FBG as a result of its limitation.

The details of numerous couplings of in-fiber grating are easily observed by a typical system: a broadband wavelength tunable light source emits a light beam into a fiber linked to the in-fiber grating, then the transmission or reflection spectrum is received by an interrogation device, such as an optical spectrum analyzer (OSA). Normally, this kind of system is spatial free. In particular, the light source and the 
interrogation device could be theoretically linked to the grating through a long distance light path (several kilometers) as a result of a very low insertion loss which occurs in the fibers. This feature brings important advantages in the use of in-fiber grating sensors as presented earlier in this paper.

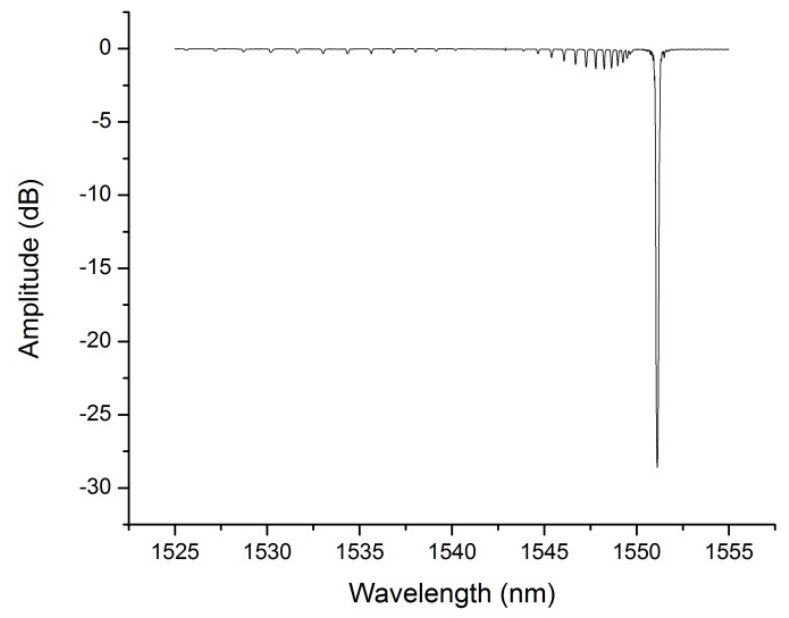

(a) Transmission spectrum of FBG

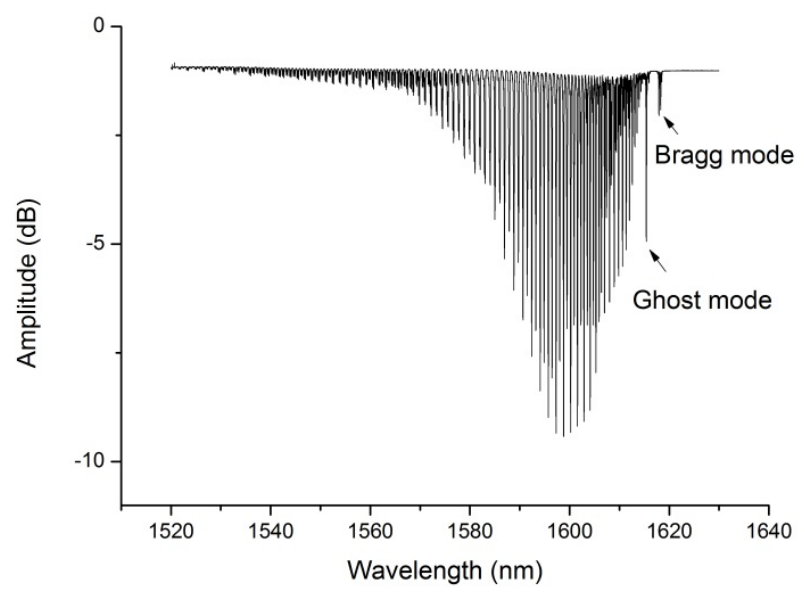

(b) Transmission spectrum of TFBG

Figure 2--2 Experimentally measured Transmission spectrum of (a) FBG and (b) TFBG 
In order to analyze the coupling mechanism of TFBG, the transmission spectra of insertion loss of two in-fiber gratings are shown in Figure2--2. One of the gratings is a normal FBG and the other one is a TFBG which has a tilted angle at $5^{\circ}$. Numerous resonances are shown in both figures. It is clear that the resonances at the longest wavelength side in both figures are the Bragg modes of the two gratings respectively (the Bragg modes are shown as a dip in the transmission spectra of both in-fiber gratings - the only strong dip in the spectrum of the normal FBG, but much weaker in the spectrum of the TFBG). Moreover, there are numerous resonances, so called the cladding modes, present at the shorter wavelength side of the Bragg modes in the transmission spectra of two in-fiber gratings. The cladding mode coupling occurs even in a perfect FBG due to the fact that the grating is limited to the core and the overlap integral is not identically zero. However, the amplitudes of cladding mode resonances are too weak in the spectrum of the normal FBG so that they are not considerable in FBG applications. However, cladding modes are enhanced significantly in the spectrum of the TFBG when the tilted angle was introduced. The amplitudes of these numerous cladding mode resonances (from the minimum peak value to the flat top of the spectrum) could be up to $-10 \mathrm{~dB}$ to -20dB (ideal state of $1 \mathrm{~cm}$-long weak tilted FBG) so they are considerable and observable. In particular, TFBG offers a multi-functional model since each cladding mode shows subtle different characteristics than each other's.

All the cladding modes locate at the shorter wavelength side since the couplings 
occur only while the phase matching condition is satisfied. According to the phase matching condition, the center wavelength of the Bragg mode of normal FBG $\lambda_{\text {Bragg }}$ is: [24]

$$
\lambda_{\text {Bragg }}=\left(n_{\text {eff,core }}+n_{\text {eff,core }}\right) \Lambda_{g}
$$

Where $n_{\text {eff,core }}$ is the effective index of the fiber core and $\Lambda_{g}$ is the period of grating. Accurately, the wavelength of the Bragg mode equals the product of the grating period and the sum of the effective index of the forward-propagating (incident) core mode and the counter-propagating (coupled) core mode, which is so-called the phase matching condition.

From equation 2-1, it is clear that the center wavelength of the Bragg mode is only subject to the wavelength of grating and the effective index of fiber core, since the forward-propagating core mode and the counter-propagating core mode are limited inside the fiber core. So the Bragg mode is insensitive to the external environment while it does not act on the physical characteristics of the grating inside the fiber core.

While the grating planes are tilted relative to the perpendicular of the fiber axis, the grating period along the fiber axis is $\Lambda=\Lambda_{g} / \cos \theta$ (relates to the tilted angle). So the center wavelength of the Bragg mode of TFBG becomes [25] :

$$
\lambda_{\text {Bragg }}=\left(n_{\text {eff,core }}+n_{\text {eff,core }}\right) \Lambda_{g} / \cos \theta
$$

The center wavelengths of cladding mode i denotes as:

$$
\lambda_{i}=\left(n_{\text {eff }, \text { core }}\left(\lambda_{i}\right)+n_{\text {eff, cladding }}\left(\lambda_{i}\right)\right) \Lambda_{g} / \cos \theta
$$


Where $n_{\text {eff,core }}\left(\lambda_{i}\right)$ is the effective index of the fiber core at the wavelength $\lambda_{i}$, $n_{\text {eff,cladding }}\left(\lambda_{i}\right)$ is the effective index of cladding mode $i$ at the wavelength $\lambda_{i}$. Since the effective index of core is always larger than that of cladding, the wavelengths of all the cladding modes are shorter than the wavelength of the Bragg mode, which is shown in Figure 2--2 Experimentally measured Transmission spectrum of (a) FBG and (b) TFBG.

The cladding mode resonance which locates at the longest wavelength is called the "ghost" mode. The ghost mode resonance always appears beside the Bragg mode and the resonance is stronger than its neighbours located at the shorter wavelength side. In reality, the ghost mode is the Bragg mode superimposed on several low order sub modes (details in Section 2.4) and it shows the similar characteristics of the Bragg mode. So the ghost mode can be seen as another "Bragg mode" which locates next to the real Bragg mode on several occasions such as the twist, strain and temperature sensors based on TFBG.

The center wavelengths of the coupling modes are important parameters in FBG and TFBG sensors. They are utilized primarily in sensing various parameters, such as strain and temperature. More specifically, the strain sensor based on FBG is utilized in particular applications in sensing structural stability and slight vibrations of bridges, dams and other buildings in various countries including the US, Canada and Europe since the 1990s.

Traditionally, FBG sensors monitor data by tracking the parameters of Bragg mode, which includes its center wavelength and amplitude value. However, compared to 
FBG sensors, the use of TFBG sensors reduces unwanted disturbances by employing the relative data of cladding modes, since each cladding mode shows independent characteristics. These unwanted disturbances are produced by several factors; firstly, in most applications, a variable quantity is monitored by tracking variations of the wavelengths or amplitudes of coupling mode resonances. Sometimes, it is hard to distinguish the real reason for these variations since the response to several parameters may cause the same variation. For example, both traditional strain and temperature sensors based on normal FBG track the wavelength shift of Bragg mode. It is hard to eliminate the interference from each other. Secondly, the disturbances may be also be caused by errors in laboratory equipment in three aspects: 1) the small displacement of light path by touching or quaking; 2) the errors occurring in normalization of the light source consisting of noise, power intensity variation and polarization displacement; 3) the setting and normalization of the interrogation system (such as an optical spectrum analyzer). Most disturbances listed above cause the move of the whole spectrum but not the relative relationship between each mode. As a result, some of these disturbances can be avoided or reduced by utilizing the relative data, such as the deviations of wavelengths and amplitudes between the resonances of Bragg mode and each cladding mode. These data can be utilized to bring a simple solution in producing a new temperature-independent strain sensor with a simplex structure, which will be introduced in next chapter. 


\subsection{Polarization dependence}

Since the introduction of the tilted angle breaks the fiber cylindrical symmetry, the grating planes reflect the incident core mode into numerous cladding modes in determined transverse directions. As a result the cladding modes have strong polarization-dependent properties [7]. With this feature, TFBG is used as an in-fiber polarizer [26], a polarization-dependent loss (PDL) equalizer [27], etc. However, polarization state parameters of TFBG also cause the splits of high order cladding mode resonances, shown in Figure 2--3. Three peaks of each cladding mode resonance are observed at around 1540nm in the figure.

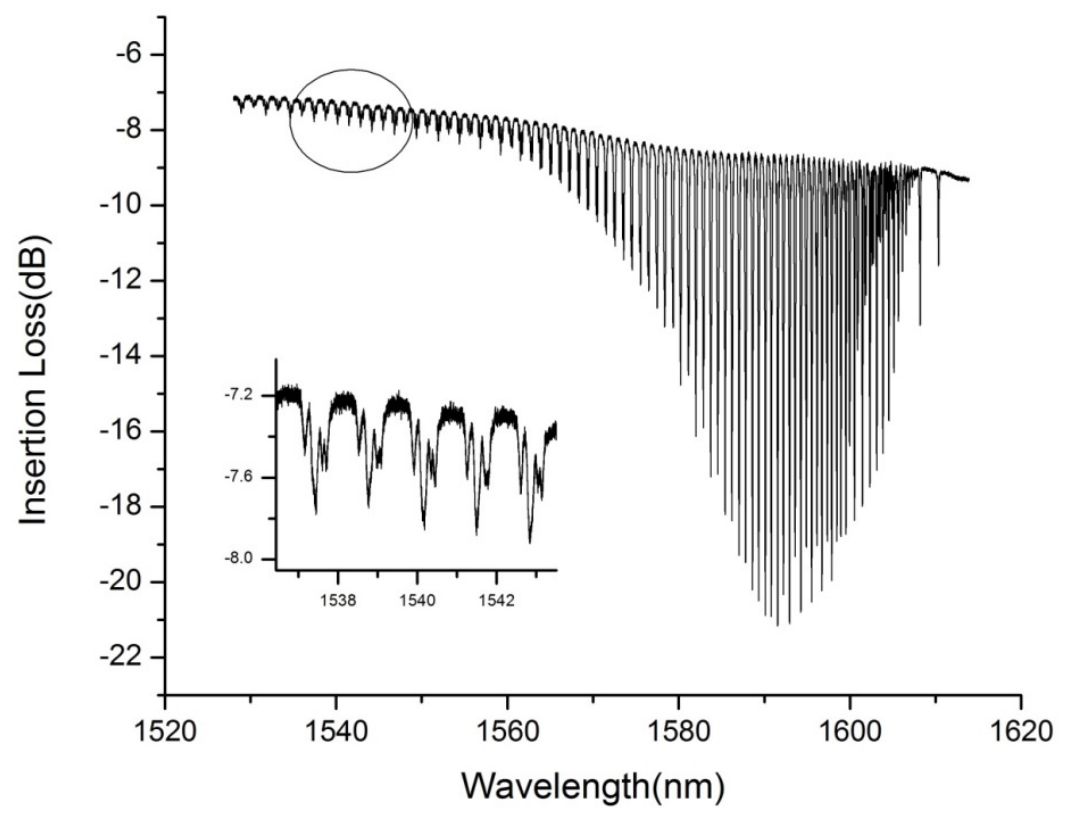

Figure 2--3 The split of high order cladding modes 
This situation happens since each coupling mode can be seen as an envelope which consists of various sub-modes with a similar effective index [28]. Actually, various sub modes consist of four kinds of electromagnetic modes: $H E_{l m}, E H_{l m} T E_{0 m}$ and $T M_{0 m}$,where $l$ is the azimuthal order, while $m$ is the radial order. $T E_{0 m}$ modes do not have axial electric field components so such modes are considered as azimuthally polarized cylindrical symmetry, while $T M_{0 m}$ modes do not have axial magnetic field components so that such modes are seen as radially polarized. In other words, $T E_{0 m}$ and $T M_{0 m}$ modes are polarization-independent. However, both $H E_{l m}$, and $E H_{l m}$ modes combine axial and transverse components and they are polarization-dependent. The numerous cladding modes of TFBG are comprised of the four types of sub modes. The most important point is that the sub modes which have similar effective indices lay in the same cladding mode resonance envelope as a result of the equation 2-3. However, sometimes the cladding mode resonances split as a result of slight differences of effective indices of sub modes which lay in the same resonance envelop.

The effective indices of the sub modes lay in a higher order cladding mode (at shorter wavelength side) show larger differences than that lay in a lower order cladding mode, so the splits usually happen in that region. Moreover, the entirety of the transmission spectrum of weakly tilted FBG $\left(\theta<10^{\circ}\right)$ appears as an inverted triangle (shown in Figure 2--2 Experimentally measured Transmission spectrum of (a) FBG and (b) TFBG). Normally, the cladding mode resonances which have maximum amplitudes 
show as smooth single-peak resonances. An explanation of the combination of various sub modes, $\mathrm{P}$ and $\mathrm{S}$ polarization states are introduced in the following section.

\subsection{1 $P$ and $S$ polarization states}

In order to analyze the polarization properties of TFBG, each cladding mode, which consists of various sub modes, can be seen as a set of two components which are perpendicular to each other. Figure 2--4 shows the profile of a TFBG, where the z-axis coincides with the fiber axis and the grating planes are tilted $\theta$ from $\mathrm{x}$-axis. Each sub mode at any polarization state can be seen as a combination which consists of two components along the $\mathrm{x}$-axis (so called p-polarized) and y-axis (s-polarized) respectively.

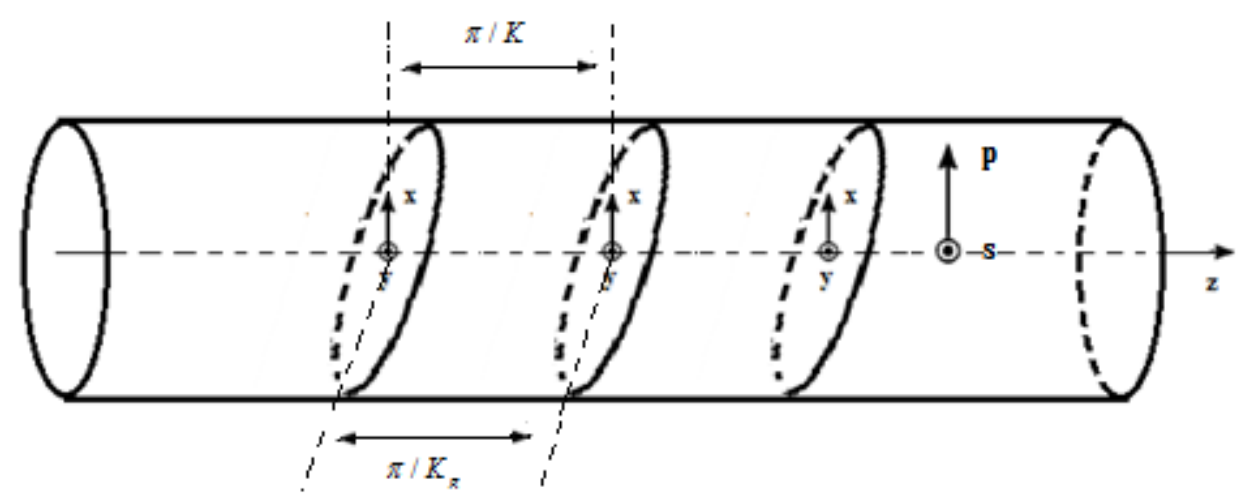

Figure 2--4 Profile of TFBG

The transmission spectra of two polarization states can be observed directly by emitting a polarized incident light into the grating, since the coupling occurs between the incident core mode and the reflected cladding modes with the same polarization state is 
much larger than the coupling occurs between the modes with different polarization states. So while the state of polarization of incident light is rotated to $\mathrm{x}$-axis or $\mathrm{y}$-axis, the cladding mode resonances of transmission spectrum show maximum amplitudes, which are higher than the amplitudes of all other polarization states. In this situation, the observed cladding modes are $\mathrm{P}$ or $\mathrm{S}$ modes. $\mathrm{P}$ and $\mathrm{S}$ modes are the components of non-polarized cladding modes which only consist of the light along the x-axis or y-axis respectively. $\mathrm{P}$ modes mainly consist of $E H_{l m}$ and $T M_{0 m}$ while $\mathrm{S}$ modes mainly consist of $H E_{l m}$ and $T E_{0 m}$. Figure 2--5 shows the transmission spectra utilizing the same TFBG as in Figure 2--3, but the perpendicular polarized incident light at P and S polarization states are emitted into the grating respectively. In Figure 2--5(a), the upper side spectrum shows the Bragg mode and ghost mode resonances of $\mathrm{S}$ polarization state, while the lower side spectrum shows the same resonances of $\mathrm{P}$ polarization state. Both of the Bragg mode and ghost mode resonances were unchanged while the polarization state of incident light rotated from $\mathrm{P}$ to $\mathrm{S}$ polarization state. It means that Bragg and ghost modes are insensitive to the polarization states. This result occurs since both the incident core mode and the reflected core mode have fiber cylindrical symmetry. However, the resonances of $\mathrm{P}$ modes and $\mathrm{S}$ modes at around $1543 \mathrm{~nm}$ show different characteristics and this feature is shown in Figure 2--5(b). For the P mode and the S mode contained in a same cladding mode resonance, several characteristics exist: The P mode resonance locates in front of (at shorter wavelength side) the S mode resonance. Meanwhile, the $\mathrm{P}$ 
mode resonance is less deep than $\mathrm{S}$ mode resonance. Moreover, the amplitude differences between two polarized modes increase, while the raise of the tilted angle or the decline of the center wavelength of the resonance (higher order mode). Based on these characteristics, $\mathrm{P}$ modes and $\mathrm{S}$ modes can be differentiated. However, the situation completely changes due to the different external environments of the grating, such as the coated TFBG, of which the spectrum shows different characteristics [29].

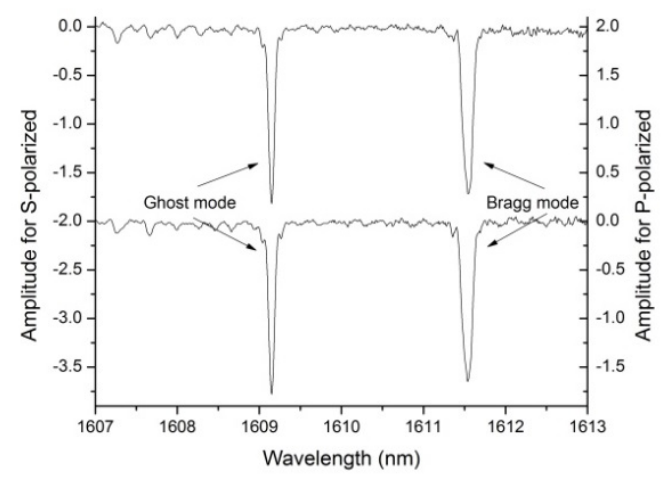

(a) Bragg and ghost modes of $\mathrm{P}$ and $\mathrm{S}$ polarization states

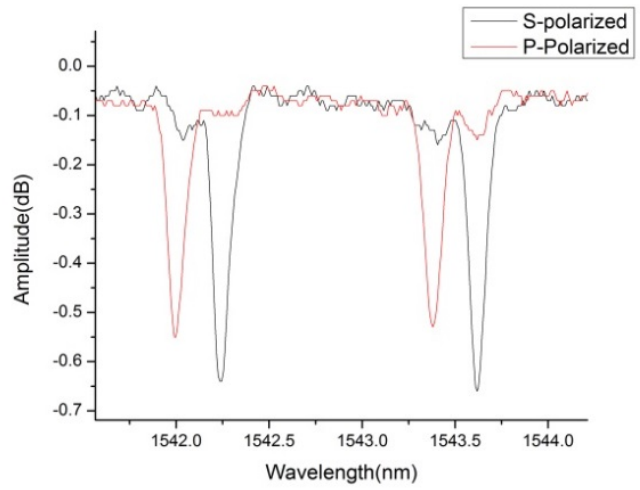

(b) Cladding modes at $1543 \mathrm{~nm}$ of $\mathrm{P}$ and $\mathrm{S}$ polarization states

Figure 2--5 Experimentally measured Transmission spectra of $\mathrm{P}$ and $\mathrm{S}$ polarization states 


\subsubsection{Coupling coefficient}

The reflectivity of cladding modes, which is shown as the amplitude of cladding resonances in the transmission spectrum, is denoted as follows:

$$
R_{i, \max }=\tanh ^{2}\left(\kappa_{i} L\right)
$$

Where $\kappa_{i}$ is the coupling coefficient between the incident core mode and the reflected cladding mode i. $L$ is the length of the grating. The coupling coefficient is given in equation 2-5, while the "DC" coupling coefficient is considered as zero [28]:

$$
\kappa_{i}=\frac{\pi n_{e f f, c o r e} \Delta n}{4 \lambda_{i} Z_{0}} \int_{0}^{2 \pi} \int_{0}^{a} \vec{E}_{\text {cor }, i}, \vec{E}_{i}^{*} \Delta \eta_{i} r d r d \phi
$$

Where $\Delta n$ represents the periodical variation of the effective index in the fiber core. $n_{\text {eff, }, \text { ore }}$ is the effective index of fiber core. $Z_{0}$ is the free space impedance. For a defined grating, the fraction in equation $2-5$ is a constant in the core and it is zero in the cladding of fiber, since $\Delta n=0$ outside core. So the radial integration is limited from 0 to the radius of the core $a . \Delta \eta_{i}=\cos \left(2 K_{g} r \cos \phi \sin \theta\right)$ represents the Non-axial variation of effective index caused by the tilted angle of TFBG. For a normal FBG, $\Delta \eta_{i}=0$ since the tilted angle $\theta$ is not exist in a FBG. $2 K_{g}=2 \pi / \Lambda_{g}$ is the grating wavevector. $\vec{E}_{\text {core }, i}$ and $\vec{E}_{i}$ presents the transverse mode fields of the incident core mode and the reflected cladding mode i respectively. $\phi$ describes the property of azimuthal caused by the quasi-three-dimensional structure of TFBG. The factor $\phi$ introduces the polarization-dependence to the coupling coefficient. 


\subsubsection{Polarization-dependence loss}

Based on the above analysis, the spectral transmission properties of TFBG strongly depend on the polarization states of the incident light. So the polarization-dependence loss (PDL), which describes properties of polarization states of TFBG, is widely utilized in polarization-relate applications, such as twist sensor. PDL is the maximum variation of the insertion loss over all possible polarization states and it is denoted in equation 2-6:

$$
P D L=10 \log _{10}\left(\frac{T_{\max }\left(\lambda_{i}\right)}{T_{\min }\left(\lambda_{i}\right)}\right)
$$

Where $T_{\max }\left(\lambda_{i}\right)$ and $T_{\min }\left(\lambda_{i}\right)$ means the maximum and minimum transmission power intensity at $\lambda_{i}$ respectively.

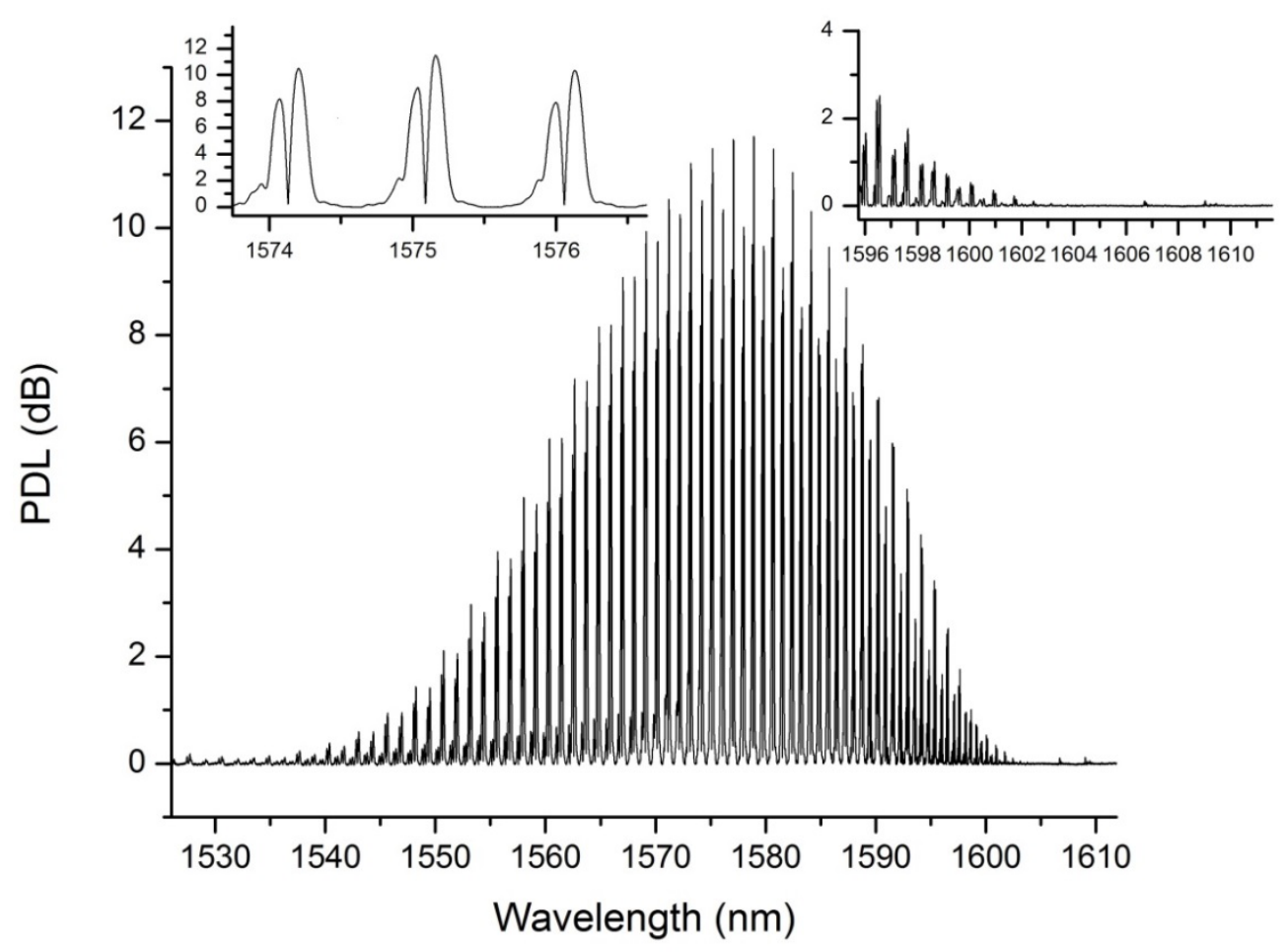

Figure 2--6 Experimentally measured PDL spectrum 
Figure 2--6 shows the PDL spectrum of a $6^{\circ}$ TFBG. The Bragg mode resonance at $1611 \mathrm{~nm}$ and the ghost mode resonance at $1609 \mathrm{~nm}$ disappear in the figure since the two modes are insensitive to the polarization states. It means the two modes have fiber cylindrical symmetry. Meanwhile, the cladding mode resonances present dual-peaks forms in the PDL spectrum. The two peaks of each cladding mode resonance are corresponding to the peaks of P and S polarized spectra. Actually, $T_{\max }\left(\lambda_{i}\right)$ and $T_{\min }\left(\lambda_{i}\right)$ in equation $2-6$ can be seen as two orthotropic polarization components along $\mathrm{x}$-axis and y-axis. (P and S polarization states) So equation 2-6 can also be written as:

$$
P D L=\left|10 \log _{10}\left(\frac{T_{p}\left(\lambda_{i}\right)}{T_{s}\left(\lambda_{i}\right)}\right)\right|
$$

Where the absolute value symbol describes that the difference of power intensity of $\mathrm{P}$ and $\mathrm{S}$ polarization states at $\lambda_{i}$ is not always a positive number or a negative number since they alternately increase and decrease periodically. 


\section{Chapter 3 Polarized Temperature-independent Strain Sensor}

\subsection{Historical perspective to strain sensor}

Strain and temperature sensors based on FBG are the most mature applications of FBG. In particular, strain sensors based on FBG were widely used in monitoring the structure of bridges, tunnels, pipelines, etc. since the 1990s. However, because both the effects of strain and temperature cause the wavelength shift of Bragg mode resonance, monitoring a single FBG cannot discriminate between the components of wavelength shifts caused by the effects of strain and temperature respectively. So most studies of strain sensors based on FBG focus on the dependence of temperature [30] [31] [32] [33]. These studies eliminated the spectral component caused by the effect of temperature disturbance by employing a dual FBG system: a relative grating only responds to temperature, while the working grating located spatially nearby monitor the effects of both strain and temperature. As a result, the spectral component of temperature was easily eliminated by analyzing the relative wavelength shift (the difference between the two gratings' Bragg mode), because the strain optic coefficient and thermo optic coefficient are wavelength dependent, but with a different wavelength dependence. Therefore measuring the Bragg wavelength of two gratings that are sufficiently far apart in wavelength can differentiate strain and temperature. However, many people tried this system but it does not work well. The dual FBG system complicated the structure. It was hard to guarantee that the spectral components of temperature on both gratings were 
totally the same. So accuracy and sensitivity were the main issues and the research orientation of these studies.

Compared to normal FBG, TFBG possess numerous cladding mode resonances present in transmission spectrum. The advantage is that relative to the Bragg mode resonance, numerous cladding modes show different features of the effects of strain and temperature. The spectral components of wavelength shifts caused by the effects of temperature show similar values, though the shifts of cladding modes caused by the effect of strain are independent. As a result, the wavelength of the Bragg mode plays the role of the relative parameter so that the effect of temperature can be eliminated by monitoring the deviation of wavelength shifts between the Bragg mode and each cladding mode. Based on this theory, a temperature independent strain sensor was designed by employing a weakly tilted FBG by Chen et al in 2006 [34]. The new design employed only one grating so the uncertainty of the differential between two gratings in the dual FBG system was avoided.

\subsection{Wavelength shift mechanism}

The center wavelength of Bragg mode resonance $\lambda_{b}$ and that of $i t h$ cladding mode resonance $\lambda_{i}$ are determined by phase-matching condition and explained in equation 2-2 and 2-3. It is clear that the center wavelengths of coupling mode resonances depend on two factors while the tilted angle $\theta$ is determined: one factor is the effective indices 
( $n_{\text {eff,core }}, n_{\text {eff,core }}\left(\lambda_{i}\right)$ and $\left.n_{\text {eff,core }}\left(\lambda_{i}\right)\right)$ and the other factor is the period of the grating $\Lambda_{g}$.

So the wavelength shifts caused by strain or temperature change can be seen as the sum

of two components caused by the two factors:

$$
\Delta \lambda=2 \Delta n_{\text {eff }}, \Lambda_{g} / \cos \theta+2 n_{e f f}, \Delta \Lambda_{g} / \cos \theta
$$

Both of the factors are affected by the axial strain change $(\Delta \varepsilon)$ and the temperature change $(\Delta T)$ respectively. Substitute equation 2-2 and 2-3 into 3-1, the wavelength shift of Bragg mode and cladding mode $\mathrm{i}$ are expressed in equation 3-2 and 3-3 while the effect of axial strain and temperature are considered separately:

$$
\begin{aligned}
& \Delta \lambda_{\text {Bragg }}=\left(\frac{2 n_{\text {eff }, \text { core }}}{\cos \theta} \frac{\partial \Lambda_{g}}{\partial \varepsilon}+\frac{2 \Lambda_{g}}{\cos \theta} \frac{\partial n_{\text {eff }, \text { core }}}{\partial \varepsilon}\right) \Delta \varepsilon+\left(\frac{2 n_{\text {eff }, \text { core }}}{\cos \theta} \frac{\partial \Lambda_{g}}{\partial T}+\frac{2 \Lambda_{g}}{\cos \theta} \frac{\partial n_{\text {eff,core }}}{\partial T}\right) \Delta T \\
& \Delta \lambda_{i}=\left(\frac{n_{\text {eff }, \text { core }}\left(\lambda_{i}\right)+n_{\text {eff }, \text { cladding }}\left(\lambda_{i}\right)}{\cos \theta} \frac{\partial \Lambda_{g}}{\partial \varepsilon}+\frac{\Lambda_{g}}{\cos \theta} \frac{\partial\left(n_{\text {eff, core }}\left(\lambda_{i}\right)+n_{\text {eff, cladding }}\left(\lambda_{i}\right)\right)}{\partial \varepsilon}\right) \Delta \varepsilon \\
& +\left(\frac{n_{\text {eff }, \text { core }}\left(\lambda_{i}\right)+n_{\text {eff, cladding }}\left(\lambda_{i}\right)}{\cos \theta} \frac{\partial \Lambda_{g}}{\partial T}+\frac{\Lambda_{g}}{\cos \theta} \frac{\partial\left(n_{\text {eff,core }}\left(\lambda_{i}\right)+n_{\text {eff }, \text { cladding }}\left(\lambda_{i}\right)\right)}{\partial T}\right) \Delta T
\end{aligned}
$$

\subsubsection{Strain dependence}

Strain is the relative stretch of the length of the fiber and it is caused by the axial tension. Strain is defined in equation 3-4:

$$
\Delta \varepsilon=\Delta l / l
$$

Where $\Delta l$ is the length variation of the fiber and $l$ is the original length of the fiber.

Meanwhile, the relative grating length change is proportional to the axial tension since the elasticity coefficient of silica fiber is a constant. So we have: 


$$
Y=\frac{F}{A \Delta \varepsilon}
$$

Where $Y$ is Young's modulus of elasticity ( $73 \mathrm{GPa}$ for the fiber), $F$ is the tension on the fiber, $A$ is the cross-section of the fiber $\left(\pi * 62.5 \mu m^{2}\right)$ and $\Delta \varepsilon$ is the strain.

While the effect of temperature is ignored $(\Delta \mathrm{T}=0)$ and the strain is fiber cylindrical symmetrical, the wavelength shift caused by strain is contributed by two components. It can be presented as follows [34]:

$$
\frac{\Delta \lambda}{\lambda}=\frac{\Delta l}{l}+\frac{\Delta n_{e f f}}{n_{e f f}}=\Delta \varepsilon+\frac{1}{n_{e f f}} \frac{\partial n_{e f f}}{\partial \varepsilon} \Delta \varepsilon=\left(1-p_{e}\right) \Delta \varepsilon
$$

Where the two fractions in equation 3-6 represents the strain sensitivity caused by the change of fiber length and the effective index respectively. $p_{e}$ denotes the effective photo-elastic coefficient and it is given in equation 3-7:

$$
p_{e}=-\frac{1}{n_{e f f}} \frac{\partial n_{e f f}}{\partial \varepsilon}
$$

And the effective index change is:

$$
\delta n_{e f f}=0.5 * n_{e f f}^{3}\left[p_{12}-v\left(p_{11}+p_{12}\right)\right] \delta \varepsilon
$$

Where $p_{11}$ and $p_{12}$ are the components of strain-optic tensor, $v$ is the Poisson's coefficient and $n_{\text {eff }}$ is the effective index.

Considering the wavelength shift of Bragg mode and cladding mode i respectively, we have:

$$
\begin{gathered}
\Delta \lambda_{\text {Bragg }}=\lambda_{\text {Bragg }}\left(1-p_{B}\right) \Delta \varepsilon \\
\Delta \lambda_{i}=\lambda_{i}\left(1-p_{i}\right) \Delta \varepsilon
\end{gathered}
$$

Where: 


$$
\begin{gathered}
p_{B}=-\frac{1}{n_{\text {eff, core }}} \frac{\partial n_{\text {eff,core }}}{\partial \varepsilon} \\
p_{i}=-\frac{1}{n_{\text {eff }, \text { core }}\left(\lambda_{i}\right)+n_{\text {eff, cladding }}\left(\lambda_{i}\right)} \frac{\partial\left(n_{\text {eff,core }}\left(\lambda_{i}\right)+n_{\text {eff,cladding }}\left(\lambda_{i}\right)\right)}{\partial \varepsilon}
\end{gathered}
$$

So the wavelength shifts of both Bragg mode and cladding mode i are proportional to the strain applied to TFBG.

For the Bragg mode of grating inscribed inside the core of silica fiber, we have the typical values: $p_{11}=0.113, p_{12}=0.252, v=0.16$ and $n_{\text {eff,core }}=1.482$ [24]. So we can calculate the effective photo-elastic coefficient for Bragg mode $p_{B} \approx 0.21$. So:

$$
\frac{\Delta \lambda_{\text {Bragg }}}{\lambda_{\text {Bragg }}} \approx 0.79 \Delta \varepsilon=0.79 \Delta l / l
$$

At $1610 \mathrm{~nm}$, we have:

$$
\Delta \lambda_{\text {Bragg }} \approx 1.27 \mathrm{pm} / \mu \varepsilon
$$

The effective indices of high order cladding modes are smaller than that of the Bragg mode. So based on equation 3-11 and 3-12, we have $p_{B}<p_{i}$, while the partial derivatives of effective indices of Bragg mode and all the cladding modes are considered as a similar value. Meanwhile, we have $\lambda_{B}>\lambda_{i}$. So considering equation 3-9 and 3-10, it is clear that $\Delta \lambda_{i}<\Delta \lambda_{B}$. It means the wavelength shifts of all the cladding mode resonances are smaller than that of Bragg mode resonance. The wavelength shift of each cladding mode resonance is independent to other modes. This feature can also be provided by the formula of the relative wavelength shift: 


$$
\begin{aligned}
\Delta \lambda_{B}-\Delta \lambda_{i} & =\lambda_{\text {Bragg }}\left(1-p_{B}\right) \Delta \varepsilon-\lambda_{i}\left(1-p_{i}\right) \Delta \varepsilon \\
= & {\left[\left(\lambda_{B}-\lambda_{i}\right)-\left(p_{B} \lambda_{B}-p_{i} \lambda_{i}\right)\right] \Delta \varepsilon } \\
\approx & \left(1-p_{B}\right)\left(\lambda_{B}-\lambda_{i}\right) \Delta \varepsilon \quad \text { while }: p_{B} \approx p_{i}
\end{aligned}
$$

Compared to $\lambda_{B}-\lambda_{i}$, the deviation between $p_{i}$ and $p_{B}$ is a very small factor. So $\Delta \lambda_{B}-\Delta \lambda_{i}$ can be approximate, consider while $p_{\mathrm{B}} \approx p_{i}$. As a result, equation 3-15 shows that the relative wavelength shift $\left(\Delta \lambda_{B}-\Delta \lambda_{i}\right)$ is subjected to the following two factors: the strain $(\Delta \varepsilon)$ and the distance of resonances from the Bragg mode to the cladding mode i $\left(\lambda_{B}-\lambda_{i}\right)$.

A higher order mode resonance has a smaller effective index, thus the wavelength shift that occurs by strain is smaller than that of a lower order cladding mode resonance at longer wavelength side. In other words, the high order resonance shows a "delay" response (smaller shift) to strain. The difference between the Bragg mode resonance and a high order cladding mode resonance is more sensitive (larger relative shift). Thus, when the Bragg mode is overlapped and utilized as a reference, the relative wavelength shift $\Delta \lambda_{\text {Bragg }}-\Delta \lambda_{i}$ increases while the distance between the two resonances $\lambda_{\text {Bragg }}-\lambda_{i}$ becomes larger. Figure 3--1 shows the experimental measured spectra which present the details of wavelength shifts of Bragg mode and several cladding mode resonances of a $6^{\circ}$ TFBG, of which the center wavelength of Bragg mode resonance is $1611 \mathrm{~nm}$. The solid line in the graphs represents the transmission spectrum of the grating measured with no strain, while the dashed line represents the data at the same situation but a strain was applied to the grating. Figure 3--1(a) shows the absolute wavelength shifts of Bragg mode 
and ghost mode. The center wavelengths of two modes increase $2.87 \mathrm{~nm}$ and $2.89 \mathrm{~nm}$ respectively. It is clear that the wavelength shift of ghost mode caused by strain is similar to that of Bragg mode. This feature was proved by Rahimi et al in 2009 [35].

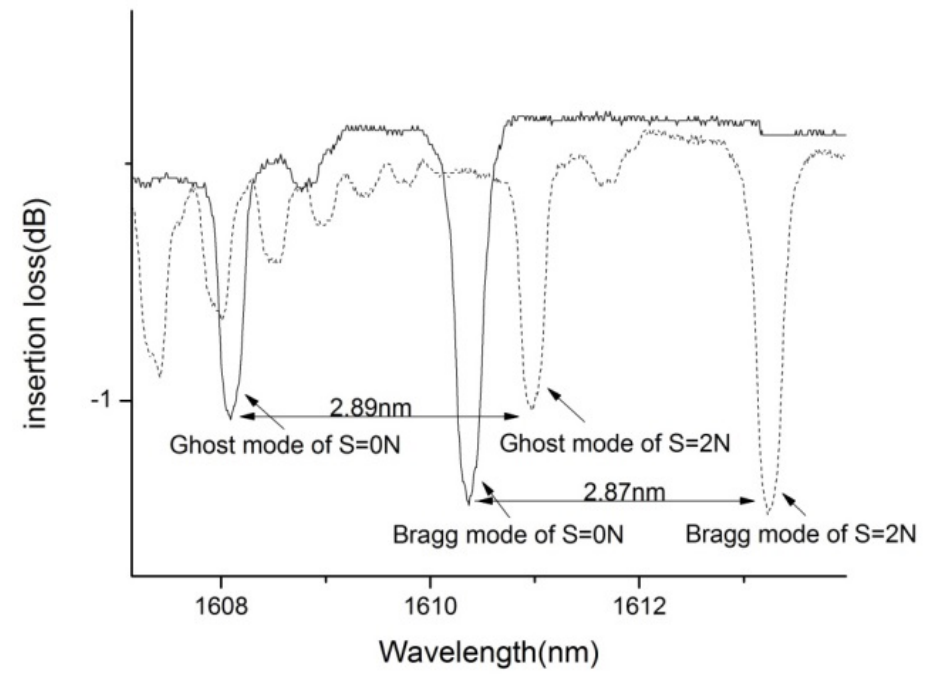

(a) The absolute shift of Bragg mode and ghost mode resonances
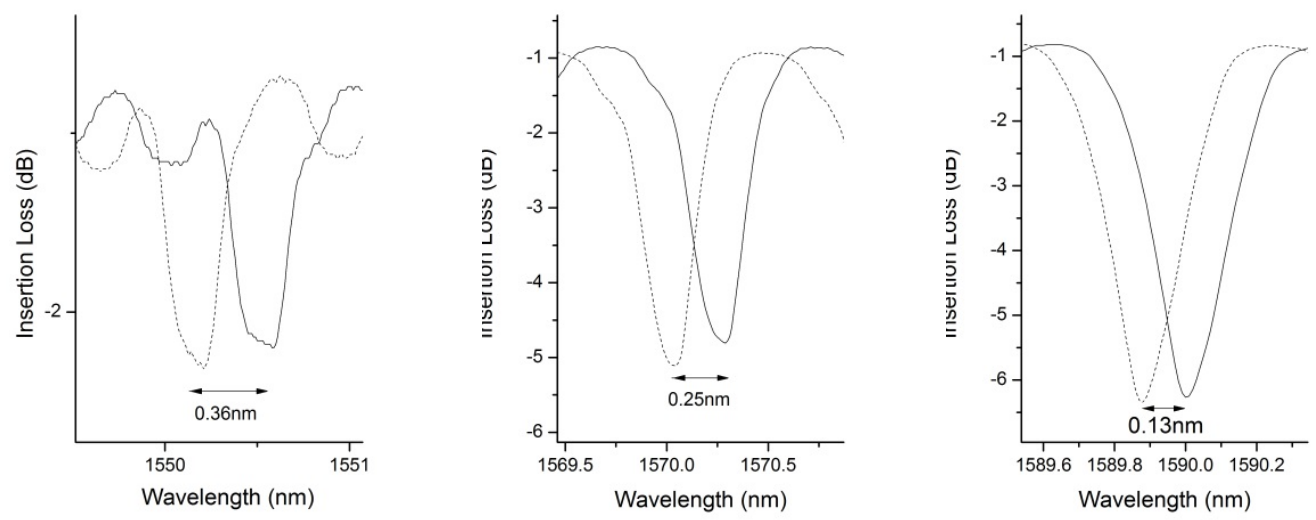

(b): relative shift at $1550 \mathrm{~nm}(\mathrm{c})$ : relative shift at $1570 \mathrm{~nm}$ (d): relative shift at $1590 \mathrm{~nm}$

Figure 3--1 Experimentally measured Wavelength shift caused by strain

The remaining three figures in Figure 3--1 show the relative wavelength shift 
$\Delta \lambda_{\text {Bragg }}-\Delta \lambda_{i}$ of three cladding mode resonances at $1550 \mathrm{~nm}, 1570 \mathrm{~nm}$ and $1590 \mathrm{~nm}$ respectively. The relative figures were obtained by overlapping the Bragg mode resonance: the center wavelength of Bragg mode resonance is shifted and overlapped at the designed wavelength of Bragg mode (at $1611 \mathrm{~nm}$ ), and the long wavelength flat top is shifted at the same level so that all spectra locate at the same level. Then we get the relative wavelength shift $\Delta \lambda_{\text {Bragg }}-\Delta \lambda_{i}$ for each cladding mode from the relate figure.

In Figure 3--1, the relative shift $\Delta \lambda_{\text {Bragg }}-\Delta \lambda_{i}$ increases from $0.13 \mathrm{~nm}$ to $0.36 \mathrm{~nm}$ while the resonances are far apart from the Bragg mode. It is clear that $\Delta \lambda_{B r a g g}-\Delta \lambda_{i}$ is proportional to $\lambda_{\text {Bragg }}-\lambda_{i}$. Compared to the absolute wavelength shift of Bragg mode resonance $(2.87 \mathrm{~nm})$, which is utilized mostly in strain sensor, the relative shifts of cladding modes are significant to be considered while $\lambda_{B r a g g}-\lambda_{i}$ is not too small. For example, the relative shift at $1550 \mathrm{~nm}$ is $0.36 \mathrm{~nm}$. It is around one in eight of the absolute shift of Bragg mode $(2.87 \mathrm{~nm})$. since each cladding mode shows independent shift, large amounts of data collected from numerous cladding mode resonances guarantee the accuracy and sensitivity by considering multiple data as an array.

\subsubsection{Temperature dependence}

Since the wavelength shift is affected by both strain and temperature and the effect of the two features is hard to differentiate, the parameter of temperature dependence is important in analyzing the characteristics of a strain sensor. The employment of deviation 
between the wavelength shifts of Bragg mode and cladding modes provides a simple-structure temperature-independent strain sensor and it is proved by Chen et al in $2006[36]$.

While only considering the effects of temperature, the equation 3-1 and 3-2 are shown as:

$$
\begin{gathered}
\Delta \lambda_{\text {Bragg }}=\left(\frac{2 n_{\text {eff,core }}}{\cos \theta} \frac{\partial \Lambda}{\partial T}+\frac{2 \Lambda}{\cos \theta} \frac{\partial n_{\text {eff,core }}}{\partial T}\right) \Delta T \\
\Delta \lambda_{i}=\left(\frac{n_{\text {eff }, \text { core }}\left(\lambda_{i}\right)+n_{\text {eff, cladding }}\left(\lambda_{i}\right)}{\cos \theta} \frac{\partial \Lambda}{\partial T}+\frac{\Lambda}{\cos \theta} \frac{\partial\left(n_{\text {eff,core }}\left(\lambda_{i}\right)+n_{\text {eff }, \text { cladding }}\left(\lambda_{i}\right)\right)}{\partial T}\right) \Delta T
\end{gathered}
$$

So the differential wavelength shift between Bragg mode and cladding mode $\mathrm{i}$ is:

$$
\begin{gathered}
\Delta \lambda_{\text {Bragg }}-\Delta \lambda_{i}=\left(\frac{2 n_{\text {eff,core }}-n_{\text {eff,core }}\left(\lambda_{i}\right)-n_{\text {eff,cladding }}\left(\lambda_{i}\right)}{\cos \theta} \frac{\partial \Lambda}{\partial T}\right. \\
\left.+\frac{\Lambda}{\cos \theta} \frac{\partial\left(2 n_{\text {eff,core }}-n_{\text {eff,core }}\left(\lambda_{i}\right)-n_{\text {eff,cladding }}\left(\lambda_{i}\right)\right)}{\partial T}\right) \Delta T
\end{gathered}
$$

For Bragg mode of a grating distributed inside the core of a silica fiber, the right side of equation 3-13 can be written as the combination of two factors:

$$
\frac{\Delta \lambda_{B}}{\lambda_{B}}=(\alpha+\xi) \Delta T
$$

Where $\xi=\frac{1}{n_{\text {eff,core }}} \frac{\Delta n_{\text {eff,core }}}{\Delta T}$ is the thermo-optic coefficient which indicates the change of effective index caused by temperature change $\alpha=\frac{1}{\Lambda} \frac{\Delta \Lambda}{\Delta T}$ is the coefficient of thermal expansion which indicates the axial length change of grating caused by temperature change. For silica fiber, $\alpha \approx 5.5 \times 10^{-7} /{ }^{\circ} \mathrm{C}, \quad \xi \approx 7 \times 10^{-6} /{ }^{\circ} \mathrm{C}$ around $150{ }^{\circ} \mathrm{C}$ and $\xi \approx 10 \times 10^{-6} /{ }^{\circ} \mathrm{C}$ around $400{ }^{\circ} \mathrm{C}$. Clearly, the change of effective index caused by 
temperature change is the mainly reason of the wavelength shifts. However, $\xi$ becomes a very small factor while the deviation between Bragg mode and cladding mode resonances are utilized. The calculated temperature dependence of the deviation value $\Delta \lambda_{b}-\Delta \lambda_{i}$ was only around $0.54 \mathrm{pm} /{ }^{\circ} \mathrm{C}$ and this value is unchanged for the entire cladding modes [36]. Compare to the absolute shift of Bragg mode caused by temperature which is around $10 \mathrm{pm} /{ }^{\circ} \mathrm{C}$ the effect of temperature could be ignored by utilizing the relative wavelength shift $\Delta \lambda_{b}-\Delta \lambda_{i}$.

\subsection{Polarization dependence of strain sensor}

It is a fact that splits occur in the cladding mode resonances since they consist of various sub-modes. These splits cause multi-peak resonances which are hard to monitor accurately. Since the small alteration of the properties of fiber (such as the length of fiber) occurs while the fiber is stretched and therefore strained, (considering the fiber is an elastic material) the splits become obvious in strain sensor experiments. Based on this, the employment of a single polarized spectrum is necessary. In particular, both $\mathrm{P}$ and $\mathrm{S}$ polarization states of weakly tilted FBG exhibit the maximum amplitudes of cladding modes so the two polarization states can be easily tracked.

Figure 3--2 shows the transmission spectra of $\mathrm{P}$ and $\mathrm{S}$ polarization states of a $6^{\circ}$ TFBG under no strain. An OSA scans the transmission spectra continually every one minute. The recorded wavelength range of spectra locates from $1528 \mathrm{~nm}$ to $1614 \mathrm{~nm}$ based 
on the bandwidth of the light source. The dashed line in the figure is the P-polarized transmission spectrum and the solid line describes the S-polarized transmission spectrum. The Bragg mode resonances of both $\mathrm{P}$ and $\mathrm{S}$ polarization states locate at $1611.5 \mathrm{~nm}$. Meanwhile, the center wavelength of ghost mode resonances is $1609.2 \mathrm{~nm}$. The cladding mode with maximum amplitude appears at around $1578 \mathrm{~nm}$ and the amplitude of $\mathrm{P}$ and S polarization states of that mode are $7.35 \mathrm{~dB}$ and $8.01 \mathrm{~dB}$ respectively. The entire spectra of $\mathrm{P}$ and $\mathrm{S}$ polarization states show similar shapes since the tilted angle of the grating is weak. $\left(6^{\circ}\right)$

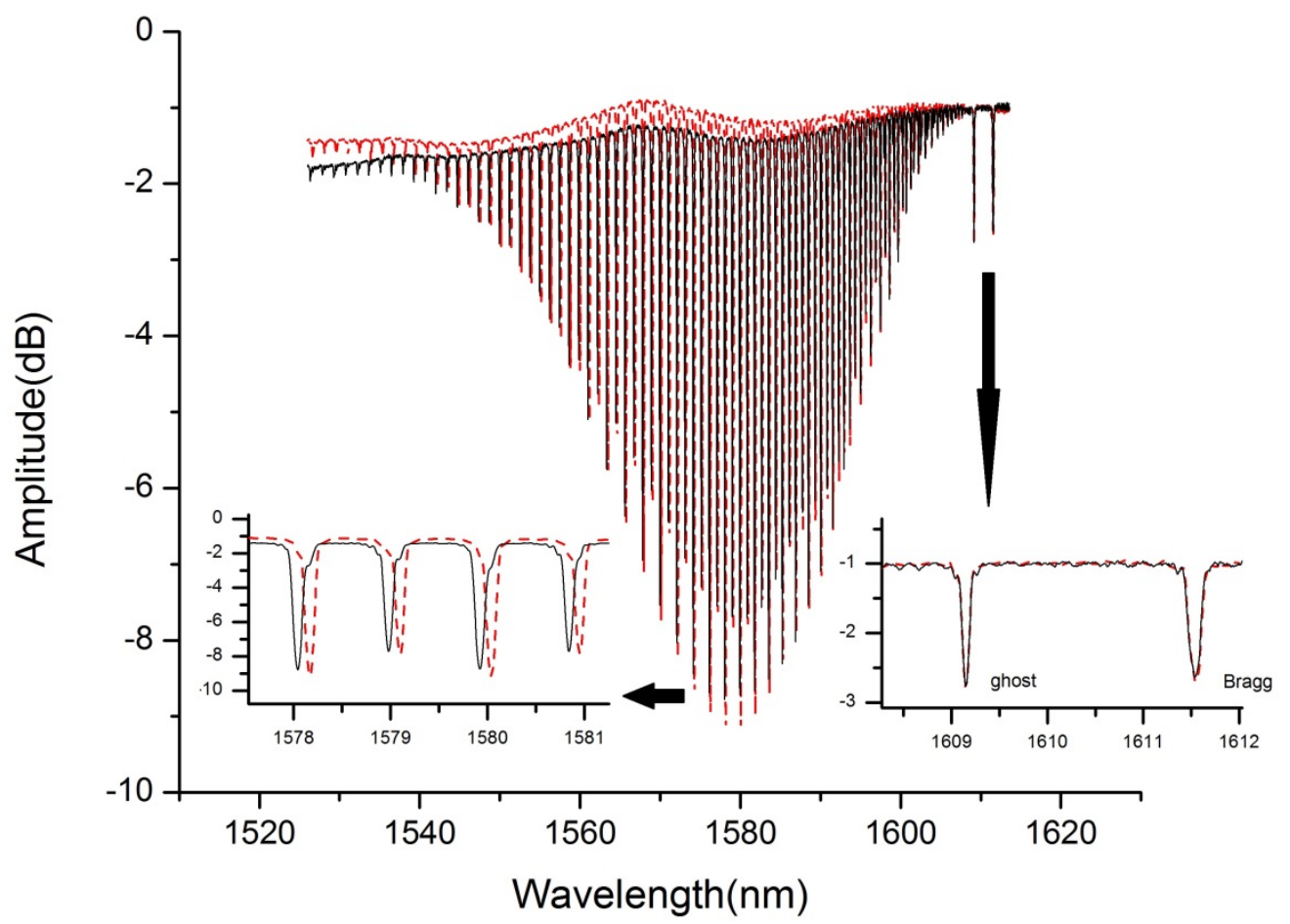

Figure 3--2 $\mathrm{P}$ and $\mathrm{S}$ polarized transmission spectrum 
In previous work by Chen et al in 2006 [34], the non-polarized spectra were utilized. As was shown, strong splits caused by the amplitude change of sub-modes and small alterations of fiber gave rise to the confused wavelength shifts of the low order modes (locates near Bragg mode) and the dual-peaks of the high order modes (located far away from Bragg mode). As a result, it was difficult to monitor the resonances in these two regions. So the numerous cladding modes were artificially divided into three groups in Chen's work and only the group at the middle of the spectrum (between $5 \mathrm{~nm}$ and $20 \mathrm{~nm}$ from the Bragg mode resonance) shows accurate results. Fortunately, the utilization of $\mathrm{P}$ and S polarized spectra avoids this problem. Figure 3--3 shows the relative spectra of $\mathrm{P}$ and $\mathrm{S}$ polarization states near the ghost mode. The solid line and dashed line represent the transmission spectra of the $6^{\circ}$ grating while the applied strain is 0 and $2 \mathrm{~N}$ respectively. The low order cladding modes shows dual-peaks resonances until the distance between cladding mode $\mathrm{i}$ and ghost mode (at $1609.1 \mathrm{~nm}$ ) becomes larger than $7 \mathrm{~nm}$ (center wavelength is smaller than $1602 \mathrm{~nm}$ ). However, the shape deformations of cladding modes and the wavelength shifts caused by strain are reasonable until the distance decreases to $2 \mathrm{~nm}$ (center wavelength is larger than $1607 \mathrm{~nm}$ ). However, the main problem is that the relative wavelength shifts $\Delta \lambda_{b}-\Delta \lambda_{i}$ in this region are too small to be detectable. Meanwhile, they are not significant enough to be distinguished from the disturbance of temperature.

Compared to the low order cladding mode resonances, the high order cladding mode 
resonances show linear results without any splits.

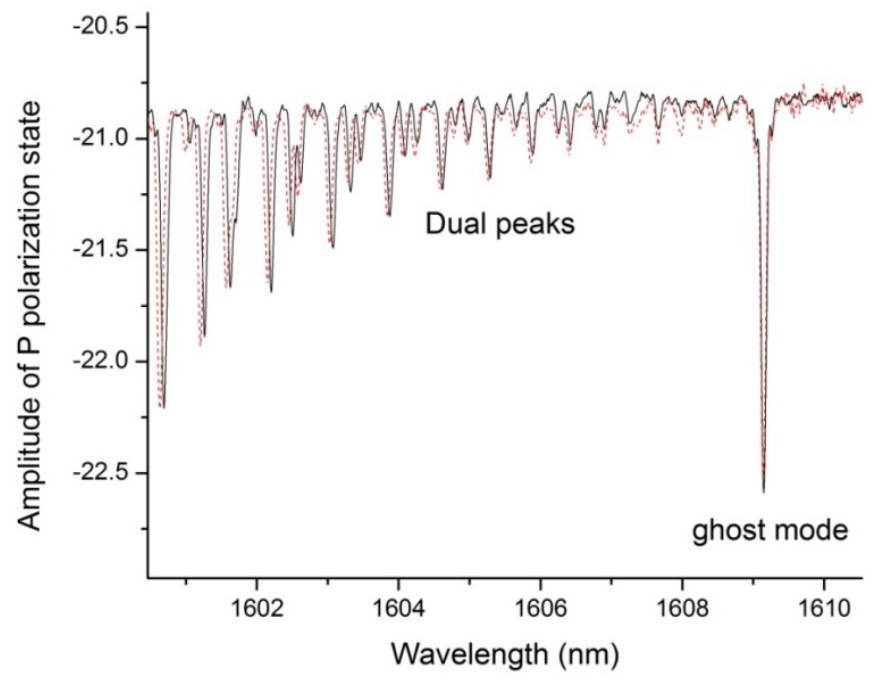

(a) P-polarized spectrum

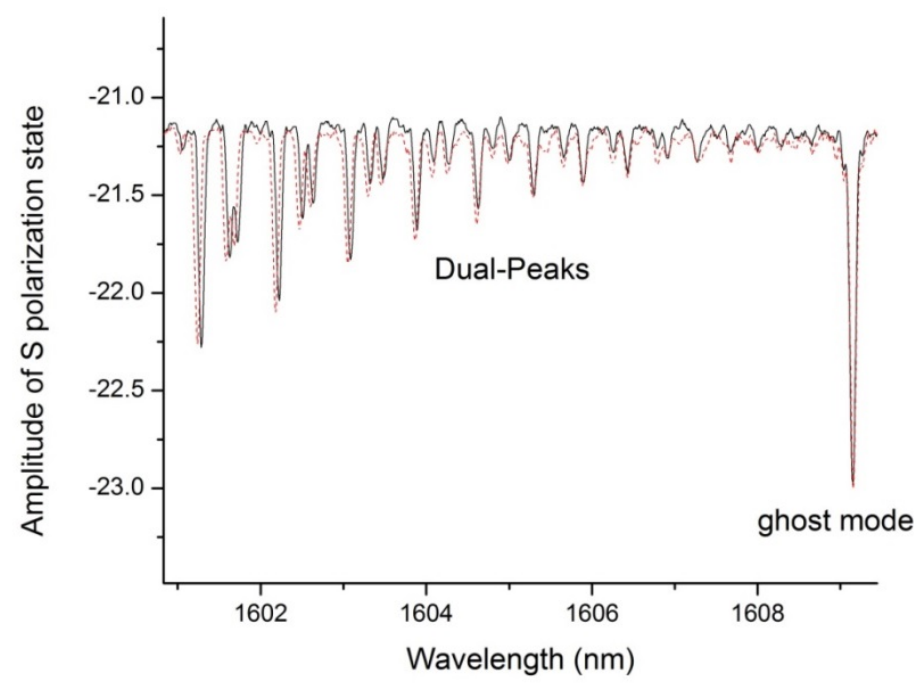

(b) S-polarized spectrum

Figure 3--3 Relative spectra of P and S polarization states near the ghost mode while the applied tension at 0 and $2 \mathrm{~N}$ respectively 


\subsection{Temperature-independent strain sensor utilizing relative polarized spectrum}

\subsubsection{Experiment set}

In order to monitor the strain response from the spectra of $\mathrm{P}$ and $\mathrm{S}$ polarization states, experiments were set up and are described below. The schematic of equipment is shown in Figure 3--4.

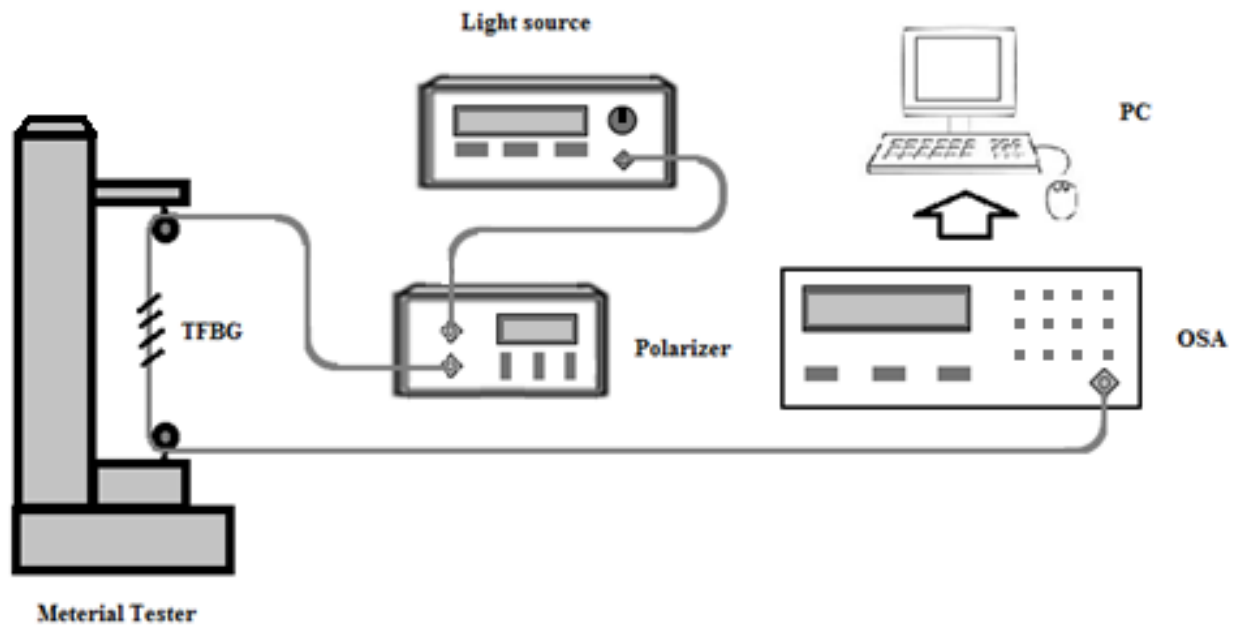

Figure 3--4 Experimental setup of strain sensor

A broadband light source provides an incident light with a wavelength from $1528 \mathrm{~nm}$ to $1614 \mathrm{~nm}$ and the light was emitted into a polarizer. Then the polarized incident light was emitted from the polarizer into a single mode optical fiber of which a $40 \mathrm{~cm}-1$ ong segment was fixed at a LF plus digital material tester produced by Lloyd Instrument. The segment of fiber was fixed vertically between two wheels. The two ends of the fiber were 
wrapped around two wheels and then clamped beside them. This manner avoided the sharp bend of fiber while it was held firmly by the clamps. The wheel at upper side could be moved vertically while the wheel at lower side was fixed on the base. The tester monitored the tension of the upper side wheel, which was the same tension applied to the $40 \mathrm{~cm}$-long fiber between two wheels. A 1 cm-long TFBG has been written inside the core of the fiber. The tilted angle of the grating was $6^{\circ}$ and the TFBG was placed at the center of the fiber between two wheels. After the incident light propagated through the TFBG, the forward-propagating light was interrogated and demodulated by an optical spectrum analyzer (OSA) to monitor the transmission spectrum. Finally, the transmission spectrum was read and recorded by a PC. In order to obtain the P and S polarization states, the polarization states of incident light was rotated by setting the polarization of the polarizer. Then the $\mathrm{P}$ and $\mathrm{S}$ polarized spectra were recorded while the maximum amplitude of cladding mode resonances was found. 


\subsubsection{Wavelength shift of strain sensor}

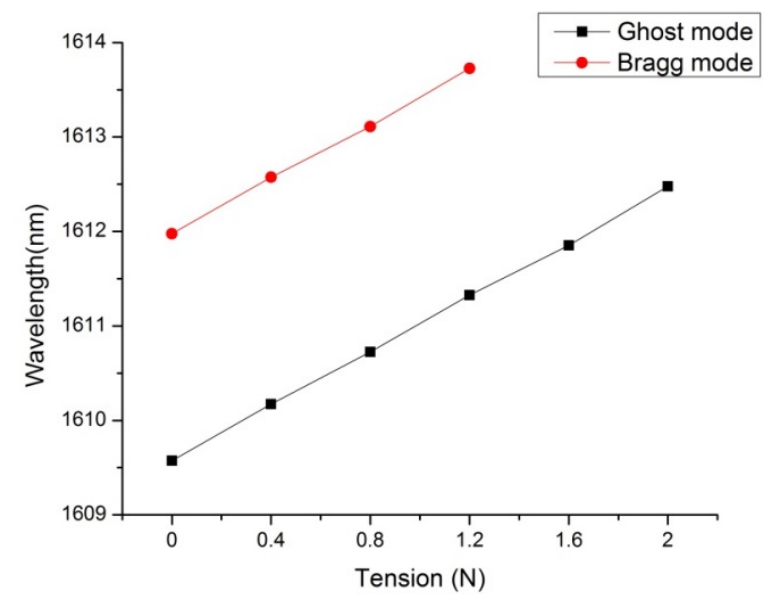

Figure 3--5 Wavelength shifts of Bragg and Ghost mode

To define the effect of strain applied to the TFBG, an increasing tension with maximum value at 2 Newton is applied by pulling the $40 \mathrm{~cm}$-long fiber. The absolute wavelength shifts of Bragg mode and ghost mode resonances are displayed in Figure 3--5. The spectra of Bragg mode at $1.6 \mathrm{~N}$ and $2 \mathrm{~N}$ are missed since the wavelength of Bragg mode increases and becomes out of the range of the OSA $(1614 \mathrm{~nm})$ while a larger tension is applied to the fiber. However, the Bragg mode and ghost mode show a similar trend $\left(\Delta \lambda_{g}=\Delta \lambda_{b}\right)$ so we can plot the relative spectrum by overlapping the ghost mode. The wavelength shift of ghost mode linearly increases from 0 to $2.9 \mathrm{~nm}$, at $2 \mathrm{~N}$.

Figure 3--6 shows the relative wavelength shifts of cladding mode resonances at $\mathrm{P}$ and S polarization states while strain is applied to the fiber. The ghost mode explained above is utilized as the reference and it is overlapped at 1610nm (collected center 
wavelengths of Bragg mode shown in Figure 3--5 are also overlapped at $1612.367 \mathrm{~nm}$ at the same time).

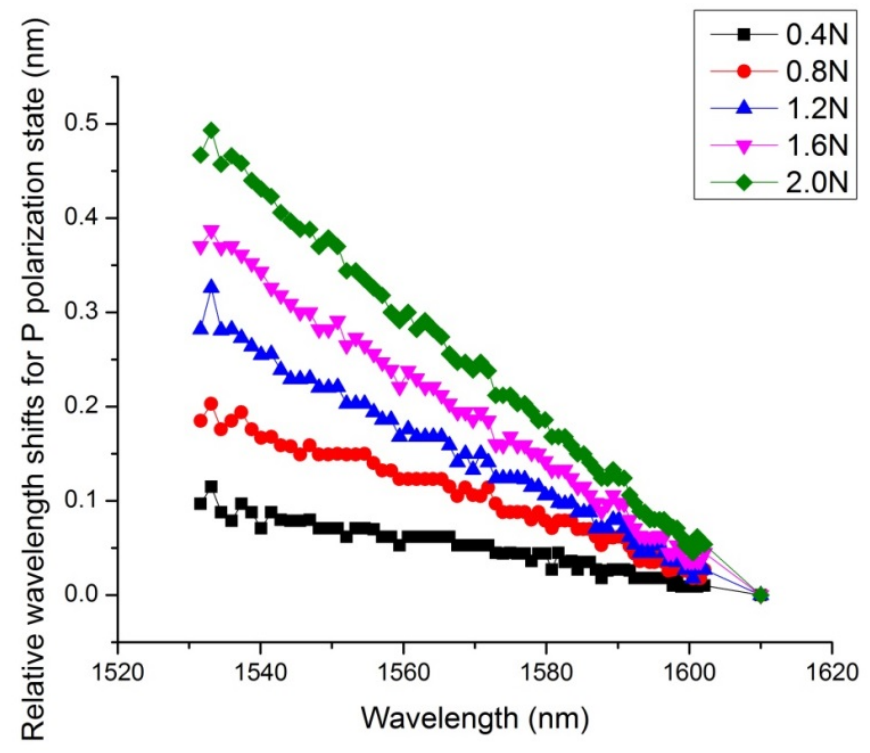

(a) P-polarized

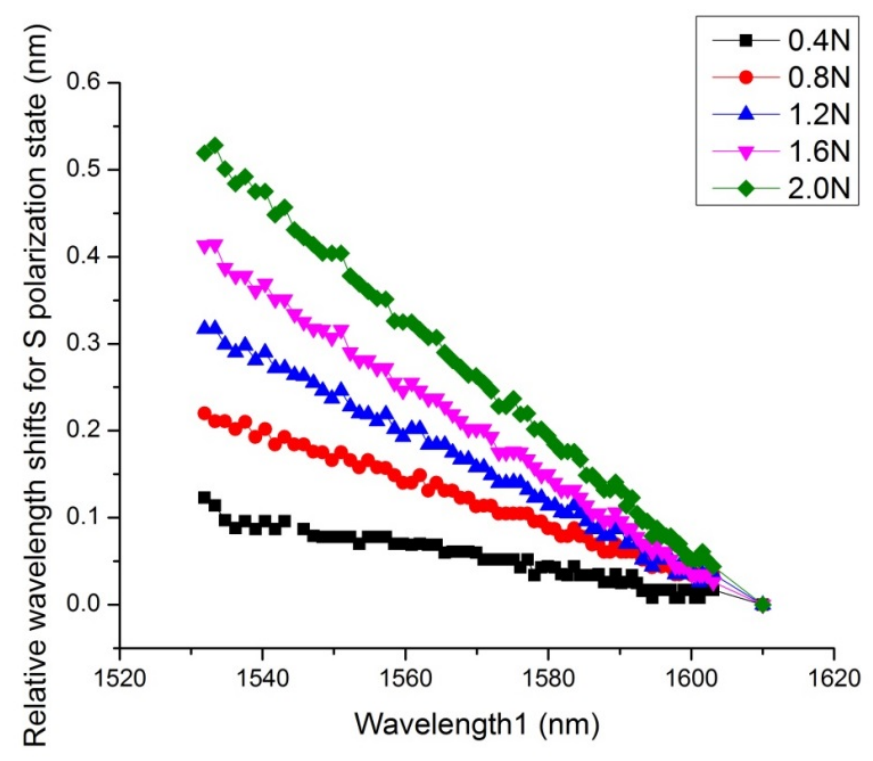

(b) S-polarized

Figure 3--6 Relative wavelength shifts of $\mathrm{P}$ and $\mathrm{S}$ polarization states 
Figure 3--6 clearly shows that for both $\mathrm{P}$ and $\mathrm{S}$ polarization states, the relative wavelength shifts $\left(\Delta \lambda_{b}-\Delta \lambda_{i}\right)$ are linearly proportional to the distance between the Bragg and cladding mode resonances $\left(\lambda_{b}-\lambda_{i}\right)$. Meanwhile, the relative wavelength shifts are influenced by the strain applied to TFBG. The wavelength shift of Bragg mode $\Delta \lambda_{b}$ is utilized to display the response to strain (explained in equation 3-13).

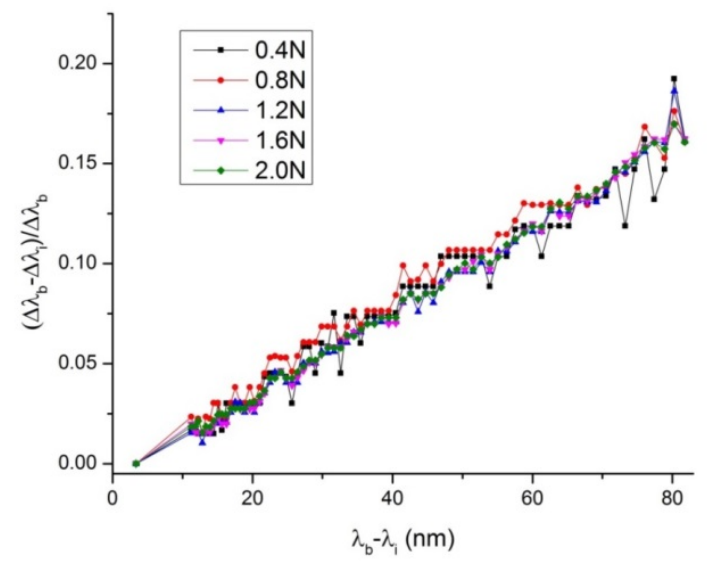

(a) P-polarized

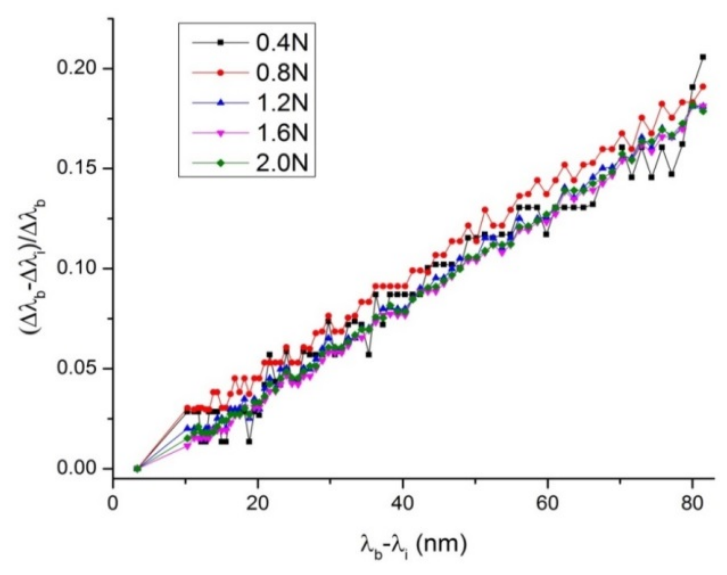

(b) S-polarized

Figure 3--7 Trends of relative wavelength shifts depend on the distance between the Bragg (ghost) mode and the cladding modes 
In order to intuitively explain how the relative wavelength shifts respond to strain, Figure 3--7 shows the trends of the ratio of relative shifts of cladding modes resonances $\left(\Delta \lambda_{b}-\Delta \lambda_{i}\right)$ and the absolute shift of ghost mode $\left(\Delta \lambda_{g}\right.$, which is same as $\left.\Delta \lambda_{b}\right)$ while the resonances are far away from the Bragg mode $\left(\lambda_{b}-\lambda_{i}\right.$, where $\left.\lambda_{b}=1612.367 \mathrm{~nm}\right)$

Different oblique lines explained the trends of the radio $\left(\Delta \lambda_{b}-\Delta \lambda_{i}\right) / \Delta \lambda_{b}$ under different tensions. Clearly, the oblique lines are overlapped in both figures for P and $\mathrm{S}$ polarization states. This means that the ratio $\left(\Delta \lambda_{b}-\Delta \lambda_{i}\right) / \Delta \lambda_{b}$ only relates to $\lambda_{b}-\lambda_{i}$ and the ratio remains unchanged while the strain applied to the fiber changes. The relative wavelength shifts $\Delta \lambda_{b}-\Delta \lambda_{i}$ show a similar trend in the wavelength shift of Bragg mode $\Delta \lambda_{b}$ while the distance $\lambda_{b}-\lambda_{i}$ is determined. Linearly fitting the oblique lines which represent the tension at 2N in Figure3-7(a) and (b), I get Figure 3--8: 


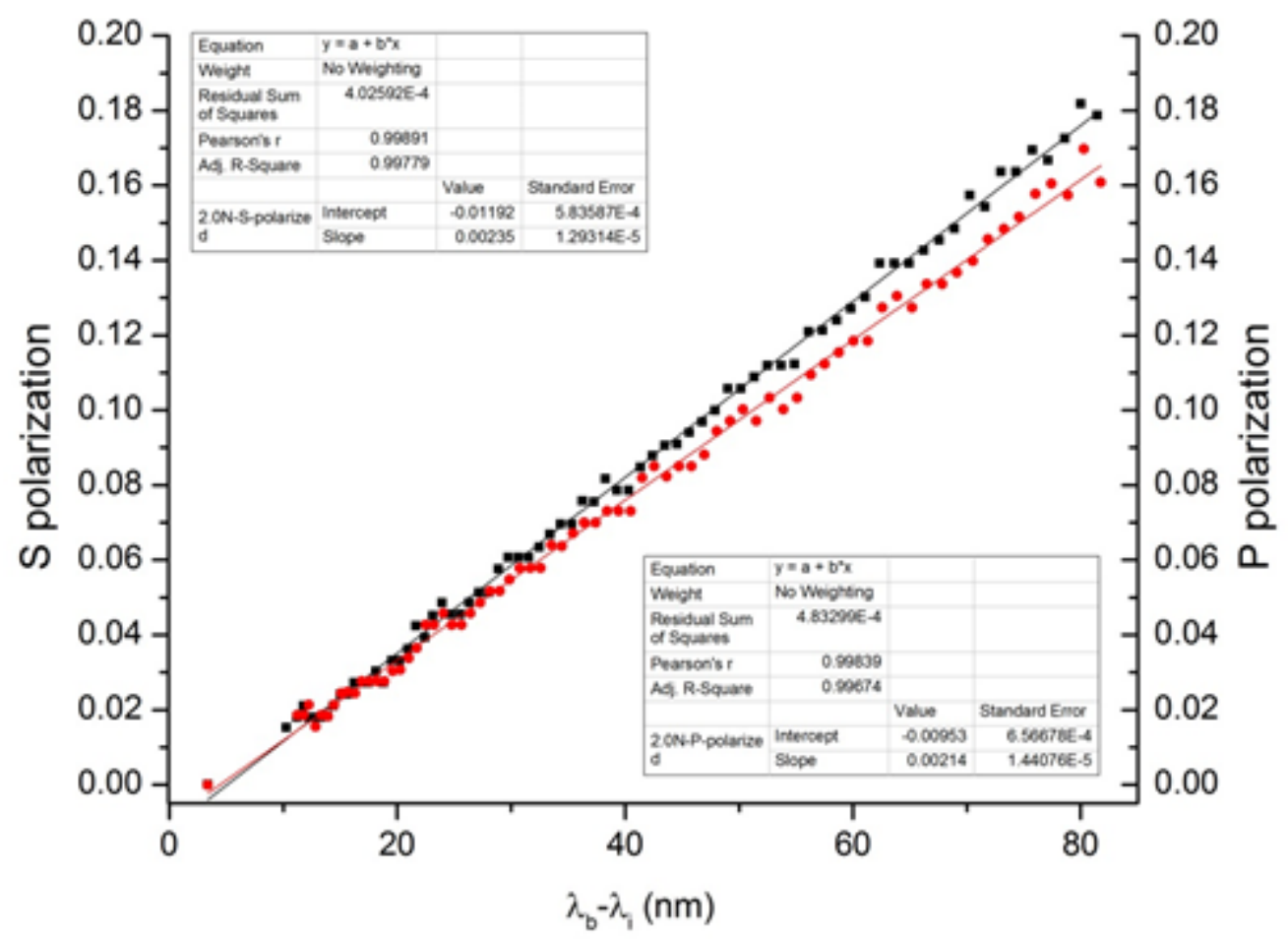

Figure 3--8 the fitted line of $\left(\Delta \lambda_{b}-\Delta \lambda_{i}\right) / \Delta \lambda_{b}$

The fitted lines of $\mathrm{P}$ and $\mathrm{S}$ polarized ratio $\left(\Delta \lambda_{b}-\Delta \lambda_{i}\right) / \Delta \lambda_{b}$ are:

$$
\begin{aligned}
& y=0.00214 x-0.00953(\mathrm{~nm}) \text { for P polarization state } \\
& y=0.00235 x-0.01192(\mathrm{~nm}) \text { for S polarization state }
\end{aligned}
$$

Where $\mathrm{y}$ is $\left(\Delta \lambda_{b}-\Delta \lambda_{i}\right) / \Delta \lambda_{b}$ and $\mathrm{x}$ represents $\lambda_{b}-\lambda_{i}$.considering equation3-9, we have:

$$
\begin{aligned}
& \left(\Delta \lambda_{b}-\Delta \lambda_{i}\right)_{p}=\left[0.00214 \times\left(\lambda_{b}-\lambda_{i}\right)-0.00953\right] \lambda_{b}\left(1-p_{b}\right) \Delta \varepsilon \\
& \left(\Delta \lambda_{b}-\Delta \lambda_{i}\right)_{s}=\left[0.00235 \times\left(\lambda_{b}-\lambda_{i}\right)-0.01192\right] \lambda_{b}\left(1-p_{b}\right) \Delta \varepsilon
\end{aligned}
$$

The fitted lines represent how the relative wavelength shift $\Delta \lambda_{b}-\Delta \lambda_{i}$ responses to 
the strain $\Delta \varepsilon . \lambda_{b}$ and $p_{b}$ are constants while the TFBG is determined. The factor $\lambda_{b}-\lambda_{i}$ describes the spectral position of the selected resonance. While the particular cladding mode $\mathrm{i}$ is selected, $\Delta \lambda_{b}-\Delta \lambda_{i}$ is proportional to $\Delta \varepsilon$. Compare equation 3-22 to 3-23; the $\mathrm{S}$ polarization state is slightly more sensitive to strain than the $\mathrm{P}$ polarization state. While the monitored cladding mode resonance departed 1nm from the Bragg mode, the relative wavelength shift $\Delta \lambda_{b}-\Delta \lambda_{i}$ of $\mathrm{P}$ polarization state increases $0.214 \%$ of the Bragg mode shift $\Delta \lambda_{b}$.At the same time, the relative wavelength shift increases $0.235 \%$ of the Bragg mode shift for $\mathrm{S}$ polarization state. Moreover, the small intercepts in equation 3-20 and 3-21 occurs because the small difference between $p_{b}$ and $p_{i}$ (explained before in equation3-15). However, the intercepts do not exist while $\lambda_{b}-\lambda_{i}$ approaches zero because $p_{i}$ approaches $p_{e}$ in that region; the fitted lines are actually curves with very small curvature.

Figure 3--9 shows the ratio $\left(\Delta \lambda_{b}-\Delta \lambda_{i}\right) / \Delta \lambda_{b}$ versus different tensions while the selected cladding mode resonances locate at $1530 \mathrm{~nm}, 1550 \mathrm{~nm}, 1570 \mathrm{~nm}$ and $1590 \mathrm{~nm}$ respectively. 


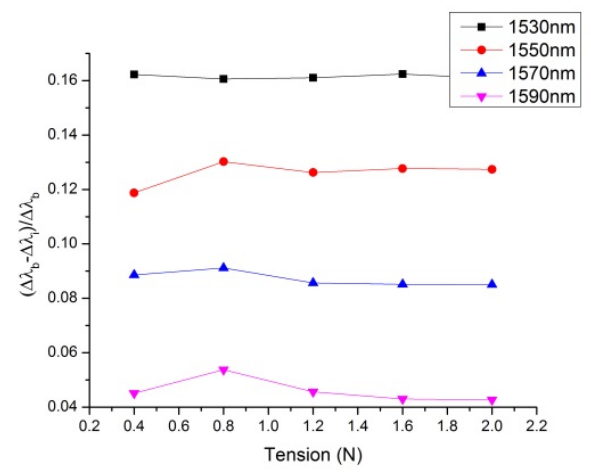

(a) P-polarized

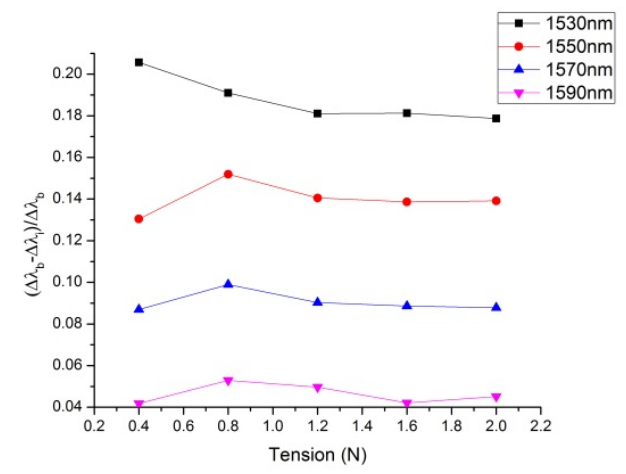

(b) S-polarized

Figure 3--9 The ratio $\left(\Delta \lambda_{b}-\Delta \lambda_{i}\right) / \Delta \lambda_{b}$ at different wavelength

The relative wavelength shifts of $\mathrm{P}$ and $\mathrm{S}$ polarization states at $1550 \mathrm{~nm}$ are $12.6 \%$ and $14 \%$ of the wavelength shift of Bragg mode $\Delta \lambda_{b}$ respectively, while the ratio increases to $16.1 \%$ and $18.8 \%$ of $\Delta \lambda_{b}$ at $1530 \mathrm{~nm}$. It means while the distance $\lambda_{b}-\lambda_{i}$ is large $(>60 \mathrm{~nm})$, the relative wavelength shift $\Delta \lambda_{b}-\Delta \lambda_{i}$ is able to take the place of the Bragg mode shift $\left(\Delta \lambda_{b}\right)$ in strain sensor, although the accuracy decreases (less than one order of magnitude) at the same resolution, however, this weak point can be remedied by employing multiple data from numerous cladding modes.

\subsubsection{Amplitude variation of strain sensor}

Beside the wavelength shifts of Bragg mode and cladding mode resonances, the amplitudes (insertion loss) of cladding mode resonances also change while the strain is applied to the fiber. In order to track the amplitude variations of strain sensor, Figure 3--10 displays the amplitude variations of $\mathrm{P}$ and $\mathrm{S}$ polarized cladding modes of the same 
TFBG utilized in last section. The amplitude variations in the figure are caused by the $2 \mathrm{~N}$ tension applied to the fiber so the variations can be written as $\Delta R=R_{2 N}-R_{0 N}$, where $R$ represents the peak values of insertion loss resonances. The amplitudes of the resonances of Bragg mode and ghost mode do not change, while the amplitudes of most cladding mode resonances for both $\mathrm{P}$ and $\mathrm{S}$ polarization states increase while the strain is being applied to the fiber.

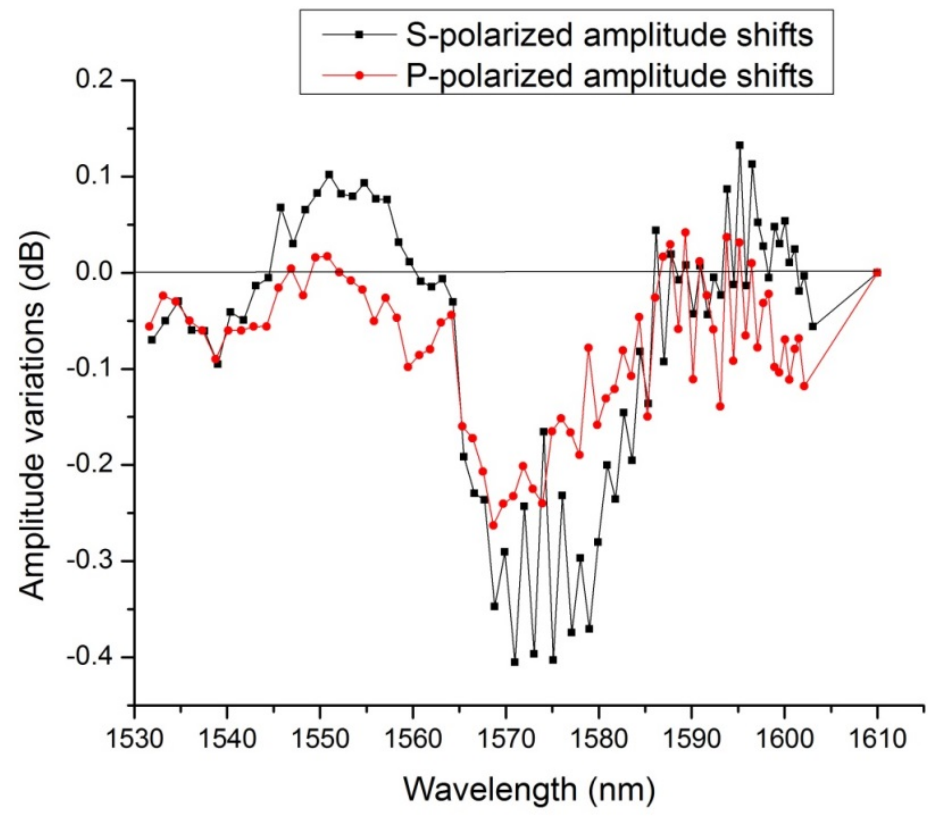

Figure 3--10 Amplitude (peak value) variations

The amplitudes increase significantly in the region between $1565 \mathrm{~nm}$ and $1585 \mathrm{~nm}$, which can be seen as the amplitude-sensitive region of strain. Meanwhile, the resonances in this region show maximum amplitudes (the bottom end of the spectra). However, the amplitude variations are small and confusedly out of the amplitude-sensitive region. The 
designed amplitudes of cladding mode resonances is calculated, based on a complex formula shown as equation 2-4 and 2-5, in which the reflectivity (amplitude of resonances) depends on the length of grating and the coupling coefficient. The amplitude variations are mainly a response to the length of grating, which is proportional to the tension applied to the fiber. The proportional relationship also applies to the amplitude variations. As a result, the analysis of the relative amplitude variation, which is shown in Figure 3--11, is necessary. The relative amplitude variation represents the ratio $\Delta R_{\text {relative }}=\Delta R / R_{0 N}$. Actually, the amplitude variations presented in Figure 3--10 describes the obtained amplitude variations in experiment, while the relative amplitude variations described in Figure 3--11 shows the extent of the variations theoretically.

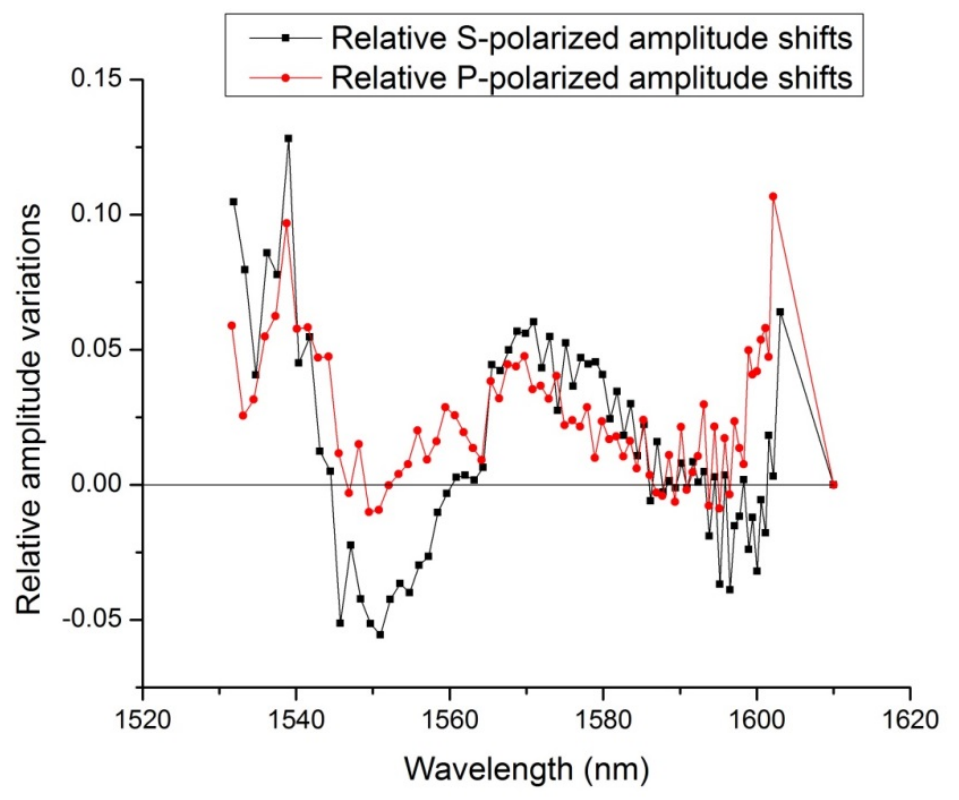

Figure 3--11 Relative amplitude variations

The obvious trends of amplitude variations are shown in Figure 3--11. The amplitudes of cladding mode resonances increase $2.7 \%$ and $4 \%$ averagely for $\mathrm{P}$ and $\mathrm{S}$ 
polarization states in the amplitude-sensitive region, in which the resonances shown maximum amplitudes of both $\mathrm{P}$ and $\mathrm{S}$ polarized spectra (the amplitude spectra of $\mathrm{P}$ and $\mathrm{S}$ polarization states are shown in Figure 3--2)

The relative amplitude variations also exhibit confused trends outside the amplitude-sensitive region. This feature, caused by the amplitude variations of flat-top of spectra, is shown in Figure 3--12. The relative $\mathrm{P}$ and $\mathrm{S}$ polarized spectra (both the wavelength and amplitude of ghost mode were overlapped) near 1550nm, 1570nm and $1590 \mathrm{~nm}$ are displayed respectively under the applied tension which increases from $0 \mathrm{~N}$ to $2 \mathrm{~N}$. Since the amplitude of ghost mode does not change while the applied tension rises, the overlapping of amplitude just ensures the whole spectra locate at the same level.

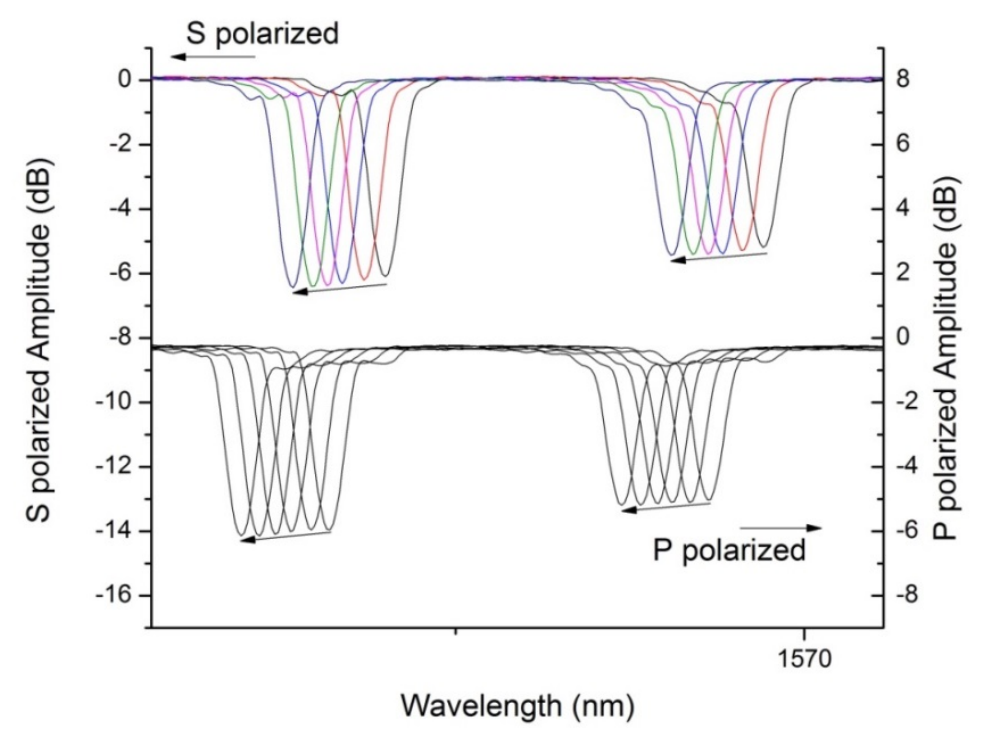

(a) Spectra at around $1570 \mathrm{~nm}$ (inside the amplitude-sensitive region) 


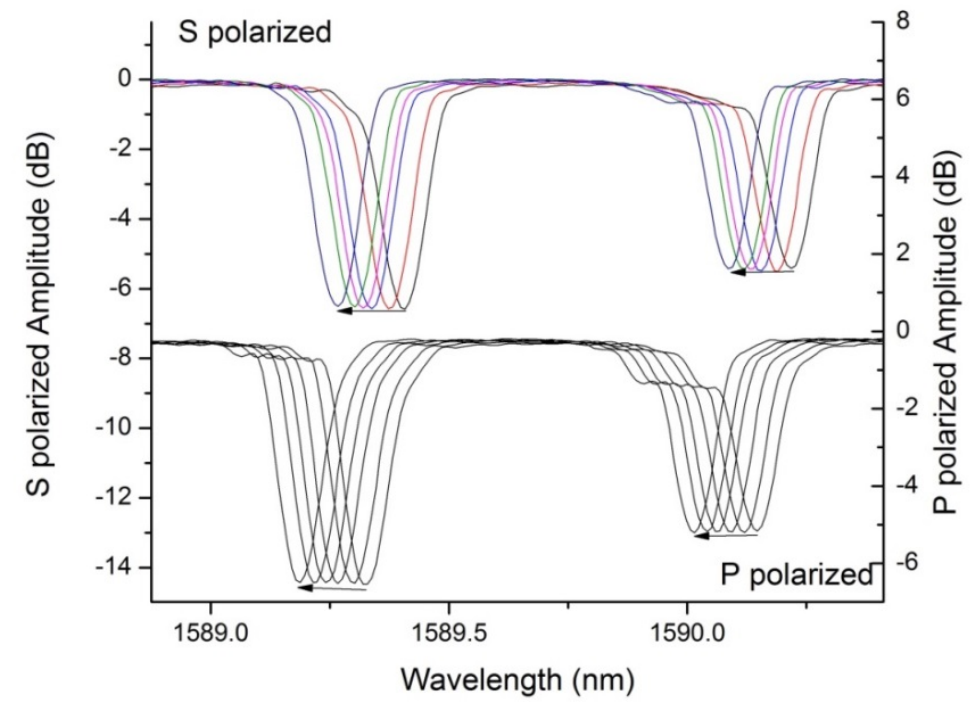

(b) Spectra at around 1590nm (outside the amplitude-sensitive region)

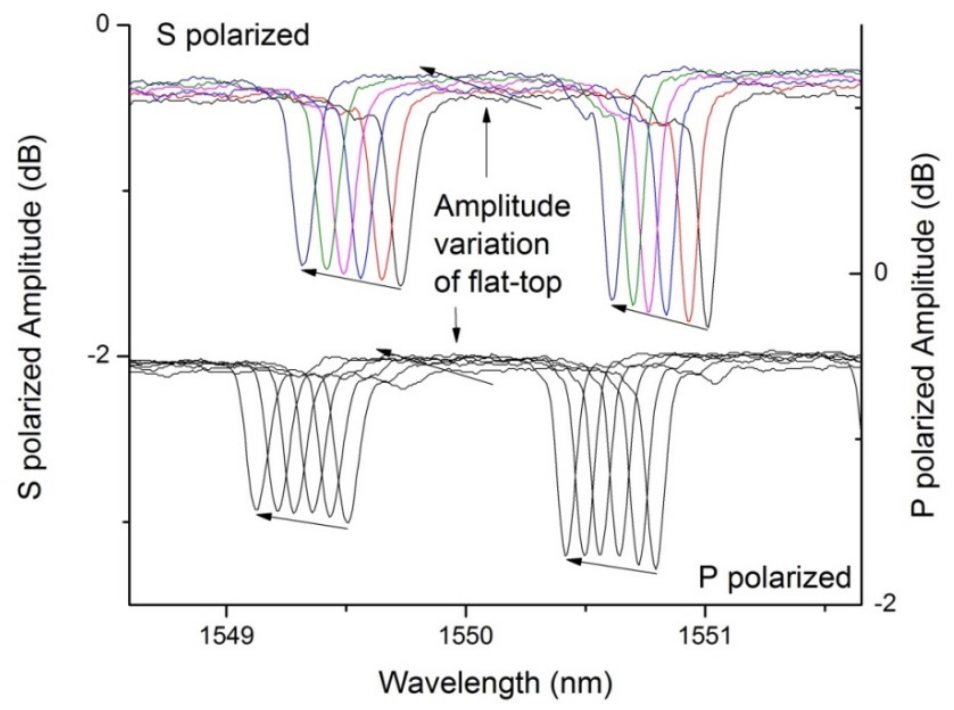

(c) Spectra at around $1550 \mathrm{~nm}$ (outside the amplitude-sensitive region) 


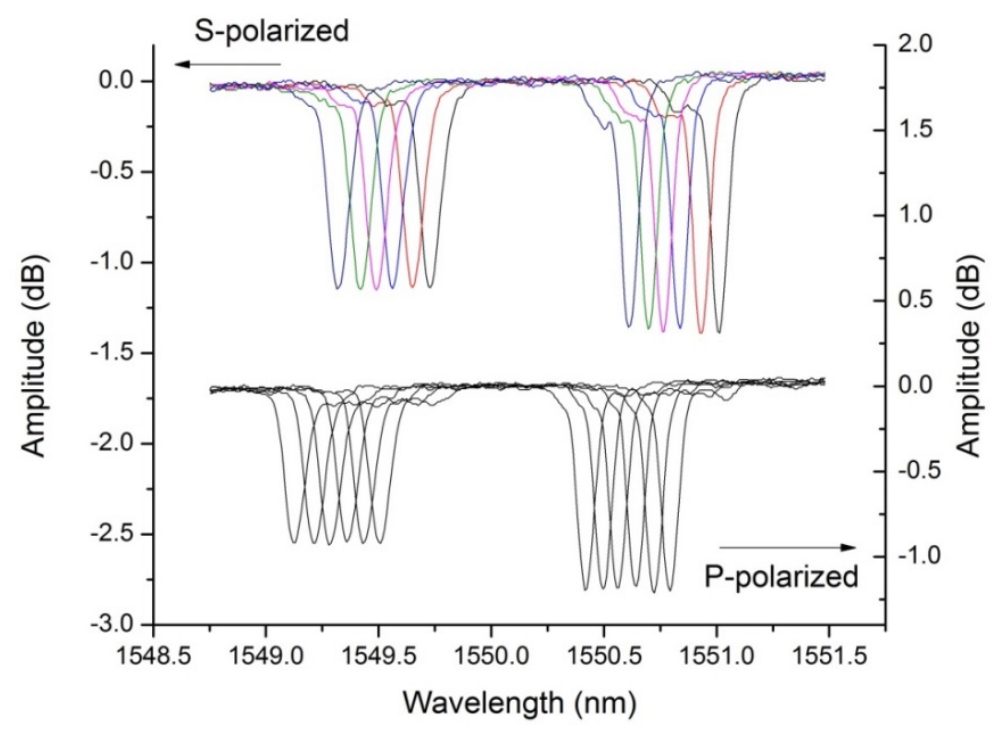

(d) Flat-top overlapped spectra at around 1550nm

Figure 3--12 The overlapped $\mathrm{P}$ and S polarized spectra at different wavelengths

Figure 3--12(a) shows the spectra at about 1570nm, which locate inside the amplitude-sensitive region. The amplitudes increase linearly while the tension increases from $0 \mathrm{~N}$ to $2 \mathrm{~N}$. However, the slight increases out of the amplitude-sensitive region is hard to figure out, For instance, the amplitudes of resonances near 1990nm stay unchanged (shown in Figure 3--12(b)) while the tension increases. So the resonances near $1590 \mathrm{~nm}$ are insensitive to strain. Moreover, an interesting appearance is shown in Figure 3--11 and Figure 3--12(c). The amplitudes of resonances near 1550nm decrease while the tension increases. This situation occurs because of the amplitude variations of flat-top. The flat-top amplitude variations occur in the region where the resonances locate far away from the Bragg mode $\left(\lambda_{b}-\lambda_{i} \geq 40 \mathrm{~nm}\right)$. This influence is difficult to eliminate while 
the absolute peak value of each of the resonances are utilized as the amplitude of the mode. However, the relative depth (from the flat-top to the peak) of the resonances near 1550nm remain stable while the tension increases, which is shown in Figure 3--12(d). For both $\mathrm{P}$ and $\mathrm{S}$ polarization states, the amplitudes of cladding mode resonances located inside the amplitude-sensitive region (1565nm-1585nm) increase while the applied tension increases, but the amplitudes of resonances located outside the amplitude-sensitive region are insensitive to the tension.

Figure 3--13 shows the relative amplitude variations of six cladding mode resonances near $1565 \mathrm{~nm}, 1575 \mathrm{~nm}$ and $1585 \mathrm{~nm}$ respectively. All the selected resonances locate inside the amplitude-sensitive region. The resonances near $1575 \mathrm{~nm}$ show significant sensitivity to the tension. Moreover, the $\mathrm{S}$ polarization state is more sensetive to strain than the $\mathrm{P}$ polarization state.

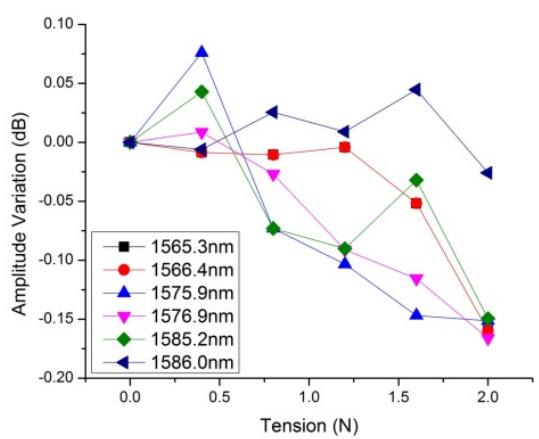

(a) P-polarization states

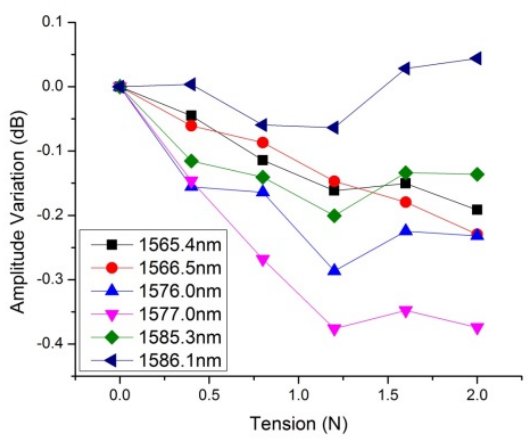

(b) S-polarization states

Figure 3--13 Amplitude variation of different resonances 


\subsection{Summary}

The theory and application of strain sensors based on TFBG is presented in this chapter. In particular, in order to reduce the disturbance caused by the effects of temperature, the relative wavelength shifts are employed to fabricate the temperature-independent strain sensor in previous work by Chen et al in 2007. However, the interference caused by the splits of cladding mode resonances becomes more serious while strain is applied to the fiber. As a result, only the resonances locate $5 \mathrm{~nm}$ to $20 \mathrm{~nm}$ from the Bragg mode shows clear response to the effects of strain. However, the employment of transmission spectra of $\mathrm{P}$ and $\mathrm{S}$ polarization states avoid the splits caused by the unwanted variations of sub-modes. So the Polarized Temperature-independent Strain Sensor was designed and introduced in section 3-3 and section 3-4. Compared to the previous work, the polarized strain sensor monitors the entire cladding mode resonances but a small region near the ghost mode (less than several nanometers). The formula of relative wavelength shifts of $\mathrm{P}$ and $\mathrm{S}$ polarization states are given in equations 3-22 and 3-23. Moreover, the amplitude of cladding mode resonances increases while the applied strain increases. However, the increases are so small that they are observable only in the amplitude-sensitive region during $1565 \mathrm{~nm}$ and $1585 \mathrm{~nm}$ for the $6^{\circ}$ TFBG of which the Bragg mode locates at $1612 \mathrm{~nm}$. Meanwhile, the amplitude variation is disturbed by various factors so only a general trend and not a linear raise was presented in section 3-4. Compared to the $\mathrm{P}$ and $\mathrm{S}$ polarization states, both the amplitude variations 
and relative wavelength shifts of S polarization state are more sensitive to the strain than that of $\mathrm{P}$ polarization state. 


\section{Chapter 4 Polarization-dependent twist sensor based on TFBG}

\subsection{Introduction to twist sensors based on various FBGs}

Twist sensor is another important application of FBG. Compared to conventional twist sensors, the twist sensor based on FBG can be flexibly embedded into engineering structures and has various advantages presented in Chapter 2. Given these advantages, several twist sensors, based on variety FBGs, were reported, such as the twist sensor based on long-period FBG [37], corrugated long-period fiber grating [6], fiber ring laser which incorporating FBGs [38], $81^{\circ}$-TFBG [39] and some special fibers [40] [41] [42]. However, these twist sensors have a variety of defects. In particular, the special structures of fibers utilized in sensing torsion angle are uneconomical and are difficult to fabricate. Thus a simple structure twist sensor based on normal FBG was announced in 2013 [43]. This twist sensor utilized a normal FBG inscribed in the core of a standard telecom single mode fiber. The relate PDL amplitude variation caused by the birefringence was monitored. The author analyzed the twist sensitivity of the difference between two peaks of PDL resonance and the sensitivity is $0.955 \mathrm{~dB} / \mathrm{rad}$. Unfortunately, it is not of significant value because the effect of birefringence is inconspicuous to FBG. 


\subsection{Theoretical analysis}

In order to provide a new high sensitive twist sensor, a twist sensor based on weakly tilted FBG with the tilted angle at $\theta$ is presented in this chapter. The TFBG subjects to the effects of the torsion angle as a result of the quasi-three-dimensional structure of TFBG. The twisted TFBG, known as the spiral grating [44], is shown in Figure 4--1.

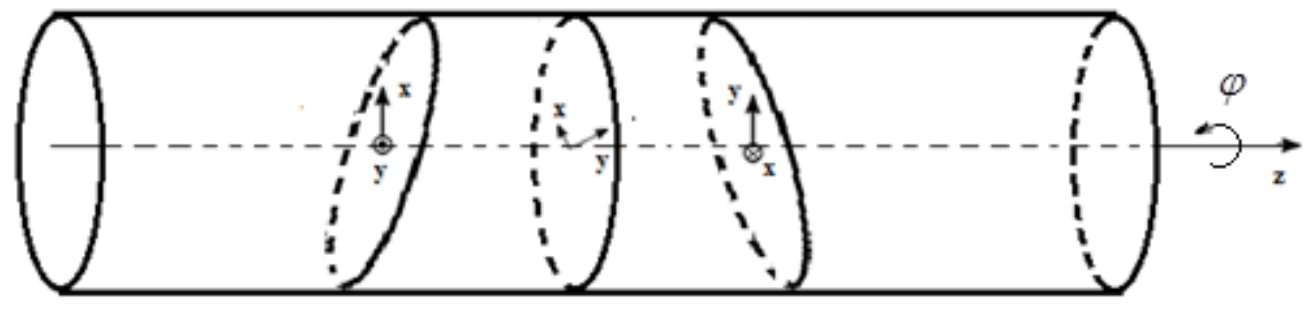

Figure 4--1 Spiral Grating

While the grating is twisted, the $\mathrm{x}-\mathrm{y}$ coordinate of grating (introduced in Chapter 2) turns along the torsion angle $\varphi$ of TFBG, where the orientation of $\mathrm{P}$ and $\mathrm{S}$ polarization states are $\mathrm{x}$-axis (tilts $\theta$ from the grating plane) and $\mathrm{y}$-axis (parallel to the grating plane) respectively. In order to analyze the effect of twist, the grating is equally divided into $N$ sections theoretically. The length of each section is: $d l=l / N$, where $l$ is the length of the TFBG. When the TFBG is twisted at angle $\varphi$, the torsion angle of each section is $d \varphi=\varphi / N$. So the Jones matrix of the twisted TFBG is [45]:

$$
M_{\text {total }}=\left(R^{-1} M R^{1}\right)\left(R^{-2} M R^{2}\right) \cdots\left(R^{-N} M R^{N}\right)
$$

Where $M$ is the Jones matrix of each section and $R$ is the matrix which describes the effect of twist of each section, and it is given in equation4-2: 


$$
R=\left[\begin{array}{cc}
\cos (d \phi) & \sin (d \phi) \\
-\sin (d \phi) & \cos (d \phi)
\end{array}\right]
$$

Equations 4-1 and 4-2 show the properties of the twisted TFBG with a unified coordinate by introducing the matrix $R$. In order to facilitate understanding, a twisted TFBG is approximated as a collection of $N$ individual grating planes. Each plane is tilted $\theta$ from the perpendicular of fiber axis $z$. Meanwhile, the transverse misalignment of two neighbouring grating planes is $d \varphi$. While an incident light is emitted into the grating, the light is coupled at each individual grating plane. In particular, the spectral properties of the grating are also approximated as the effect of the collection of the $N$ misaligned couplings at each grating plane, so the main variation of spectral properties focuses on the polarization-dependence as a result of the misalignments. In other words, the polarization-independent properties, such as the transmission insertion loss spectrum, is unchanged while TFBG is twisted, since the coupling occurs in each grating plane remain unchanged. However, the properties of insertion loss slightly change as a result of the shape distortion of grating planes.

Compared to the insertion loss spectrum, the polarization-dependent spectrum, such as PDL, is sensitive to the effects of twist and of grating. Significant variations of polarization-dependence spectrum occur based on the misalignment, which is caused by the torsion angle. This is not as a result of the birefringence but caused by the cylindrical asymmetry structure. As presented before, the coupled sub modes are highly dependent on the polarization states of incident light. The coupling between the modes with the 
same polarization states is much stronger than that of different polarization states. So P and $\mathrm{S}$ modes are severely limited to $\mathrm{x}$-axis and $\mathrm{y}$-axis respectively. For each grating plane, coupling modes are not subjected to the torsion angle. However, the coupling modes for different grating planes are transverse misaligned. So the components of $\mathrm{P}$ and $\mathrm{S}$ modes along a unified coordinate of each coupling modes are employed while the superposition is calculated.

In order to analyze the polarization-dependent properties of twisted TFBG, PDL spectrum is employed as a result of various advantages. First of all, PDL is relative data, which is simply calculated by various parameter sets, such as the difference between eigenvalues of Jones matrix or the difference between maximum and minimum loss, where maximum and minimum loss present the extreme values of all the possible polarization states at each wavelength point. Moreover, various devices, such as the optical vector analyzer produced by Luna technologies, provide the function to measure PDL spectrum directly. Then, PDL shows polarization-dependent properties from its dual-peaks form of each cladding mode. The two peaks correspondent to $\mathrm{P}$ and $\mathrm{S}$ modes respectively so comprehensive information of polarization-dependence is shown by PDL spectrum by employing the relative and absolute data from the two peaks. Moreover, the unified $\mathrm{x}$ and $\mathrm{y}$ axis which show the maximum magnitude of couplings during all the polarization states do not represent $\mathrm{P}$ and $\mathrm{S}$ polarization states respectively, but show the combination of the coupling modes at rotated polarization states, as a result of the 
misalignment of polarization states at massive grating planes. So it is difficult to figure out the orientation of $\mathrm{P}$ and $\mathrm{S}$ polarization states for the whole grating, along with the increase of the torsion angle. In other words, the $\mathrm{x}$-axis and $\mathrm{y}$-axis are hard to determine for twist sensor. Table 4--1 lists the azimuthal angles of polarized incident light at which the transmission insertion loss spectra show maximum amplitudes versus the torsion angle. As we know, the received transmission spectra should be P and S mode spectra based on the azimuthal angles explained before.

Table 4--1 Azimuthal angles versus torsion angle

\begin{tabular}{|l|l|l|l|l|}
\hline $\begin{array}{c}\text { Torsion angle } \\
\text { Of fiber } \\
\text { (degree) }\end{array}$ & $\begin{array}{c}\text { Torsion angle } \\
\text { Of grating } \\
\text { (degree) }\end{array}$ & $\begin{array}{c}\text { Azimuthal angle } \\
\text { Of P mode } \\
\text { (degree) }\end{array}$ & $\begin{array}{c}\text { Azimuthal angle } \\
\text { Of S mode } \\
\text { (degree) }\end{array}$ & $\begin{array}{c}\text { Increased } \\
\text { Azimuthal angle } \\
\text { (degree) }\end{array}$ \\
\hline 0 & 0 & 198 & 108 & None \\
\hline 120 & 13.19 & 91 & 181 & 73 \\
\hline 240 & 26.38 & 150 & 240 & 59 \\
\hline 360 & 39.57 & 206 & 116 & 56 \\
\hline 480 & 52.76 & 102 & 192 & 76 \\
\hline 600 & 65.96 & 163 & 73 & 61 \\
\hline
\end{tabular}

The first column of the table shows the torsion angle applied to a $9.1 \mathrm{~cm}$ long fiber. A $1 \mathrm{~cm}$ long TFBG is inscribed at the center of the segment of fiber. As a result, the calculated torsion angle applied to the $1 \mathrm{~cm}$ long TFBG is listed in the second column. 
Columns 3 and 4 describe the azimuthal angle applied by a polarizer at which the transmission spectra show maximum amplitudes. Obviously, the angles can be seen as the azimuthal angles of $\mathrm{P}$ and $\mathrm{S}$ modes. The azimuthal angles increase about 65 degrees while the fiber is twisted at 120 degrees, which is almost two times of the azimuthal angles. As a result, the TFBG should be placed at the center of the fiber. Moreover, the polarization of incident light has to be adjusted in each measurement since it rotates along with the torsion angle. So it is hard to define the P and S modes directly. Adjustment of the polarization states in each measurement brings large error.

To sum up, PDL spectrum is generally utilized in describing the polarization properties of various FBGs due to its advantages.

\subsection{Experiment set}

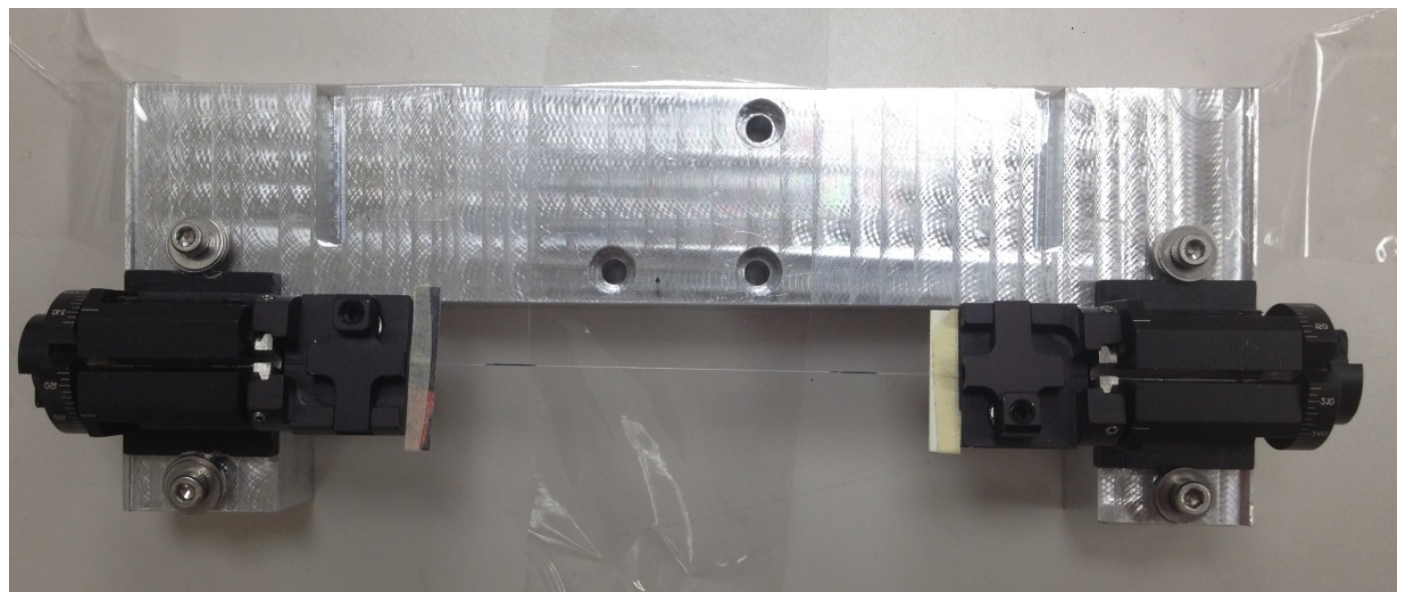

Figure 4--2 Fiber rotator

Simple experiments were set up to measure twist sensitivity of TFBG. The test $1 \mathrm{~cm}$ long TFBG was inscribed inside a standard single mode optical fiber and the center 
wavelength of Bragg mode of the TFBG locates at $1611 \mathrm{~nm}$. The tilted angle of the TFBG is 6 degree. A $9.1 \mathrm{~cm}$ long optical fiber with the TFBG positioned in the middle was clamped to a fiber rotator, which is shown in Figure 4--2. One end of the fiber was fixed to a static clamp, while the other end was fixed to a clamp which rotates along the cross section of fiber. The rotatable clamp was rotated up to $2 \mathrm{rad}$ in the experiments so the maximum torsion angle applied to the TFBG was $0.22 \mathrm{rad} / \mathrm{cm}$. SWS-OMNI-2 swept wavelength system produced by JDS Uniphase Inc. (JDSU) was employed in monitoring the transmission spectra of the TFBG. The tunable light source of the system emitted four polarized incident light (shown in Table 4--2) into the static end of the test fiber and then transmitted through the TFBG, before the receiver of the system. The incident end was determined at the static end since not only was the torsion angle applied to the TFBG, but the connected fiber beyond the rotatable clamp was also subjected to the effect of the rotate at the clamp. In order to reduce the effect of unwanted rotate to the connected fiber, the fiber connected to the rotatable clamp was straightened along the axial of the TFBG and fixed to another clamp which was placed some distance away. The details are shown in Figure 4--3(a). As well, sharp bends were avoided along the entire light path since they would cause the change of polarization states of light (for example, a sharp bend may cause a change from linear polarized light into an elliptical polarized light). 
Table 4--2 Polarization states of incident light

\begin{tabular}{|l|l|}
\hline 1 & Circular polarization \\
\hline 2 & Linear polarization at $0^{\circ}$ \\
\hline 3 & Linear polarization at $90^{\circ}$ \\
\hline 4 & Linear polarization at $-45^{\circ}$ \\
\hline
\end{tabular}

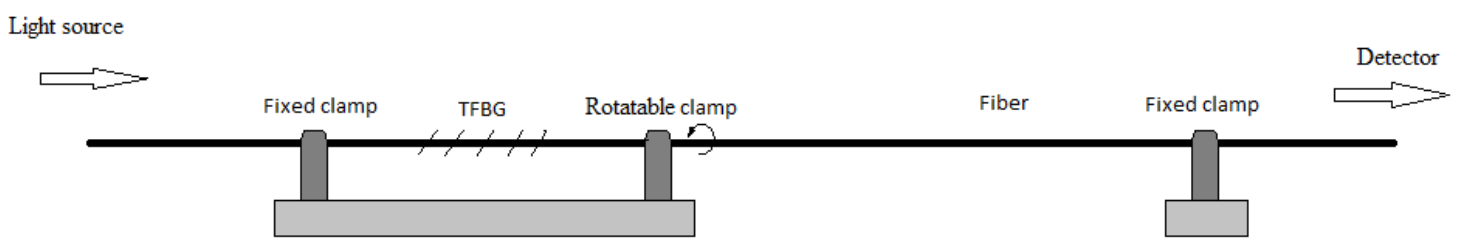

(a) Experiment set

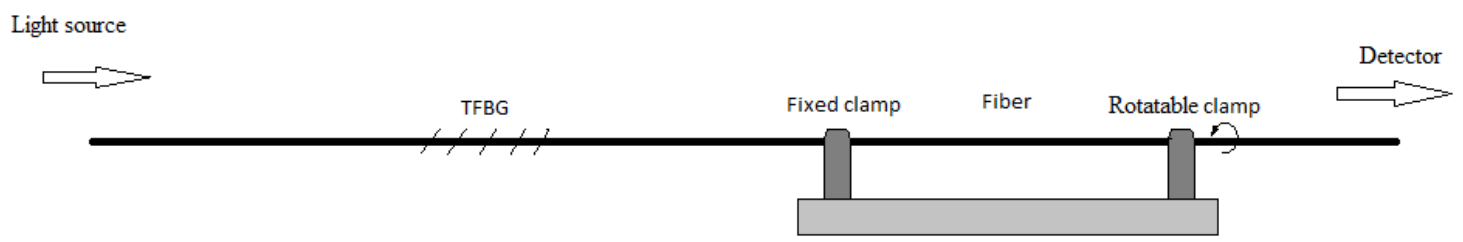

(b) Addition experiment

Figure 4--3 Experiment set 
The connected fiber was fixed in order to avoid any displacement, which would cause an obvious effect to the experiment's results. However, the unwanted rotate at the connected fiber is impossible to avoid since the light path is a loop from light source to receiver. In order to distinguish the expected experimental results from the effect of rotate on the connected fiber, an additional experiment was setup as follows and shown in Figure 4--3(b). The connections were same as described before but the TFBG was kept untwisted. The torsion angle was applied to a segment of connected fiber in order to make obvious the effect caused by the torsion angle applied to the fiber. The insertion loss spectrum remained unchanged while the fiber twisted 0.22rad/cm (shown in Figure 4--4(a)) while the amplitudes of PDL spectrum showed very small variations (shown in Figure 4--4(b)). The maximum variation was less than $0.09 \mathrm{~dB}$, where the amplitude of the resonance was around $10.3 \mathrm{~dB}$. These small variations may be caused by the torsion angle applied to the fiber. However, it may be caused by errors as well, since the errors of PDL spectrum are not as small as insertion loss spectrum (described in the next chapter). However, the additional experiment demonstrates that the effect of twisted connected fiber does not exist to the twist sensor. This confirmed that for a TFBG, the effect of torsion angle is not mainly caused by birefringence, which also exists in connected fiber. 


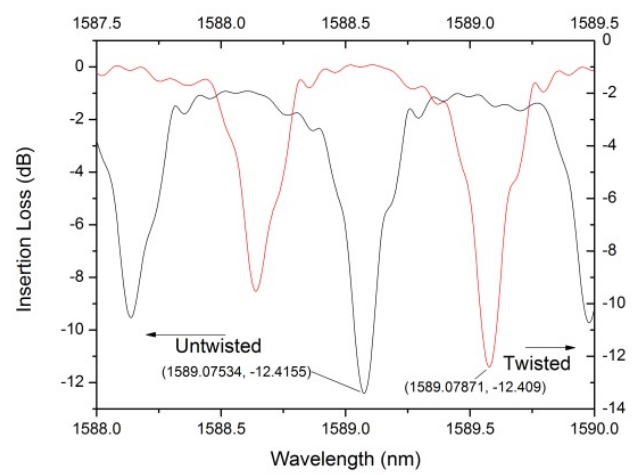

Bragg \& ghost mode

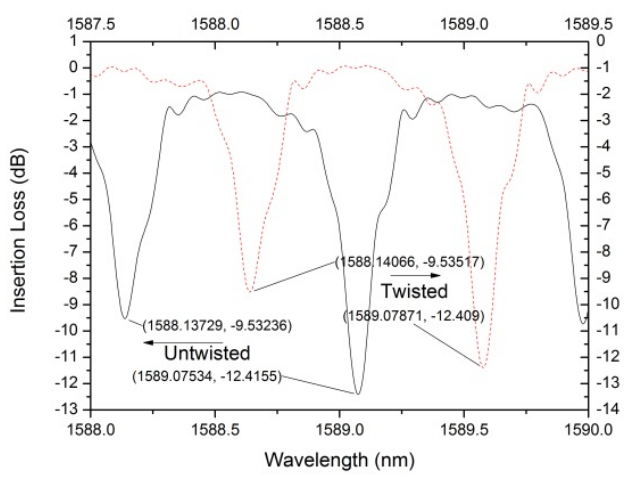

maximum cladding mode

(a) Insertion Loss

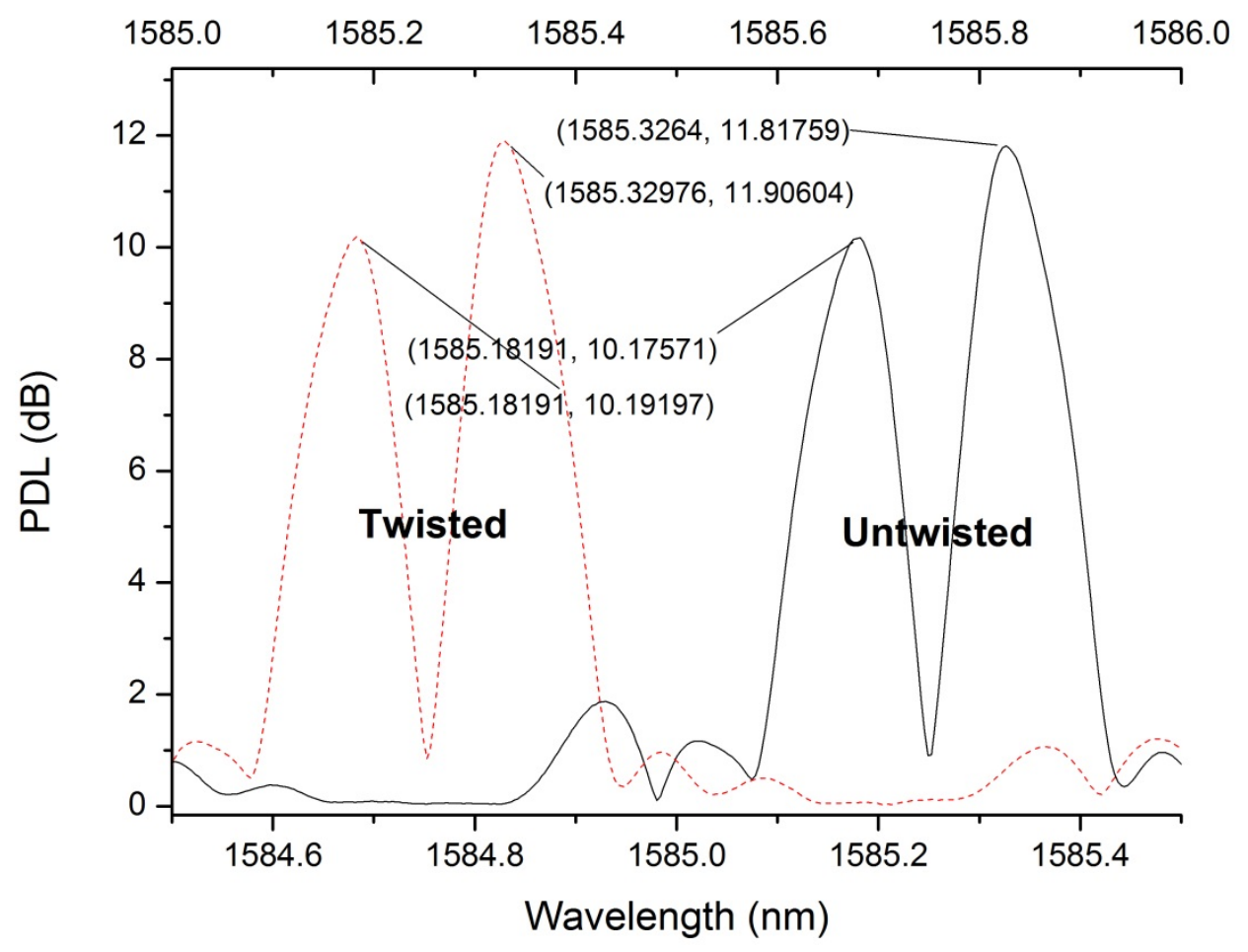

(b) PDL

Figure 4--4 Transmission spectra versus torsion angle applied to the connected fiber 


\subsection{Experimental results}

Theoretical analysis in Section 4.2 presents approximate model of a twisted TFBG while considering the TFBG is a combination of independent grating planes. However, a TFBG is spatially continuous so the torsion angle also causes the shape distortions of grating planes. As a result, the non-axial variation of effective index $\Delta \eta_{i}$ becomes a complex and unpredictable factor while calculating the coupling coefficient from equation 2-5. Fortunately, the effects caused by shape distortions are not significant. Thus, the experimental results follow the analysis presented in Section 4.2.

\subsubsection{Non-polarization properties}

Figure 4--5 shows the transmission insertion loss spectra of a $6^{\circ}$ TFBG while the TFBG is untwisted and under a torsion angle at $2.2 \mathrm{rad} / \mathrm{cm}$. The TFBG is $1 \mathrm{~cm}$ long. Two resonances, consisting of the Bragg mode and ghost mode resonances of both spectra, are presented in Figure 4--5. The two spectra are dislocated in Figure 4--5 in order to show the resonances clearly since the variations of Bragg and ghost mode resonances versus the torsion angle are very small. 


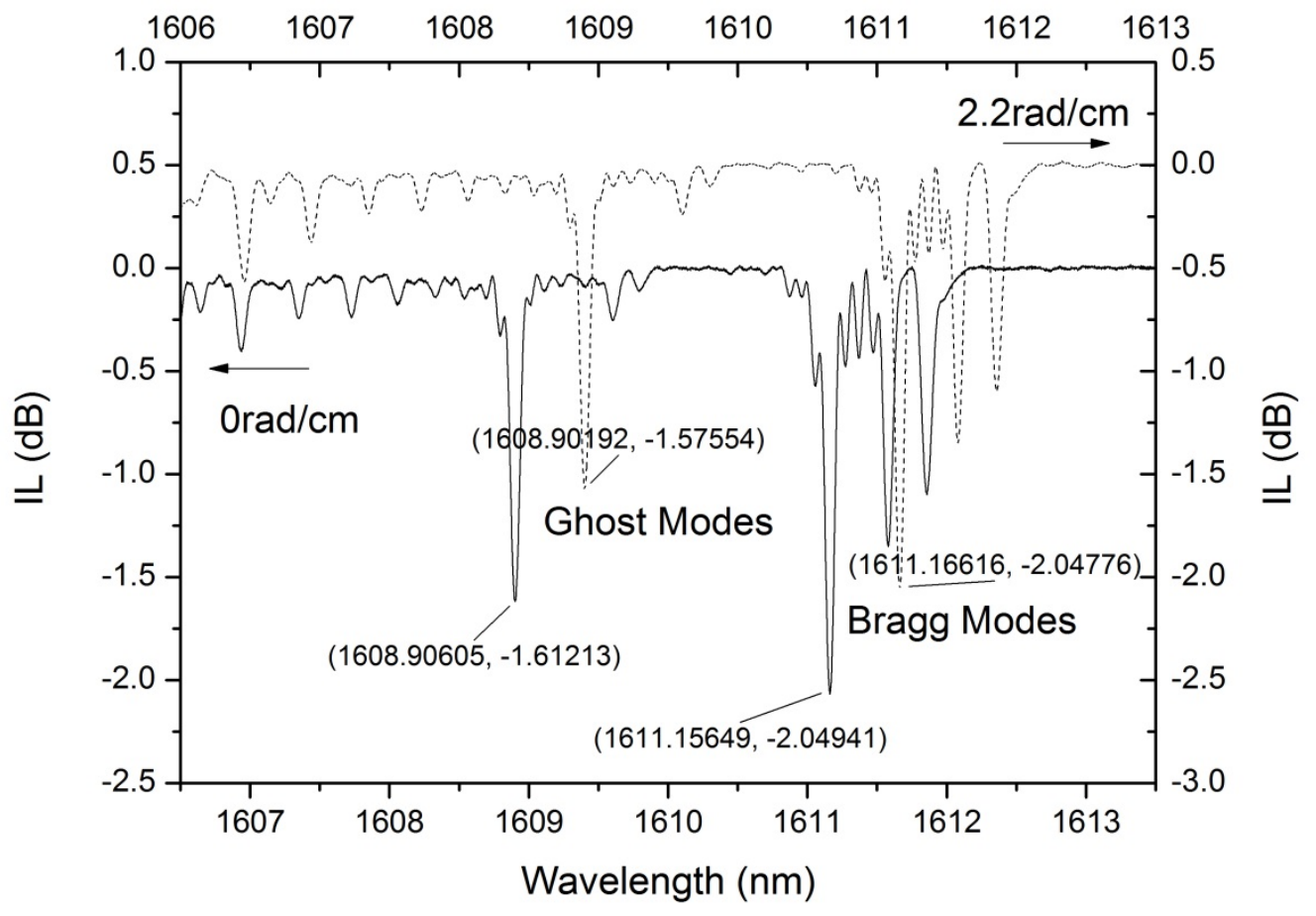

Figure 4--5 Transmission insertion loss VS Torsion angle

Table 4--3 The variations of Bragg and ghost mode resonances

\begin{tabular}{|l|l|}
\hline Wavelength shift of Bragg mode & $<0.01 \mathrm{~nm}$ \\
\hline Amplitude shift of Bragg mode & $<0.1 \%$ \\
\hline Wavelength shift of ghost mode & $<0.01 \mathrm{~nm}$ \\
\hline Amplitude shift of ghost mode & $\approx 2.3 \%$ \\
\hline
\end{tabular}

Figure 4--5 clearly shows that the Bragg mode resonance is independent of the torsion angle. Both wavelength and amplitude of the Bragg resonance remain stable 
while the TFBG is twisted, since the Bragg mode is polarization independent. Meanwhile, a small amplitude variation occurs to the ghost mode resonance. However, the variation is $2.3 \%$ of the amplitude of ghost mode at a $2.2 \mathrm{rad} / \mathrm{cm}$ torsion angle (shown in Table 4--3).

Compared to the Bragg mode, which shows insensitive properties to the torsion angle, numerous cladding mode resonances show obvious changes as a result of the torsion angle applied to TFBG, The changes are not congruent with the explanation in Section 4-2. In order to present the changes of cladding mode resonances caused by the effect of torsion angle, the wavelength shifts and relative amplitude variations (the amplitude variations over the amplitude of the resonances) are shown in Figure 4--6.

The wavelength responses of torsion angle for cladding mode resonances are too small (less than $0.01 \mathrm{~nm}$ ) to be distinguished from errors in measurements. Actually, the torsion angle does not shift the center wavelengths of cladding mode resonances as a result of the unchanged grating length and effective indices. 


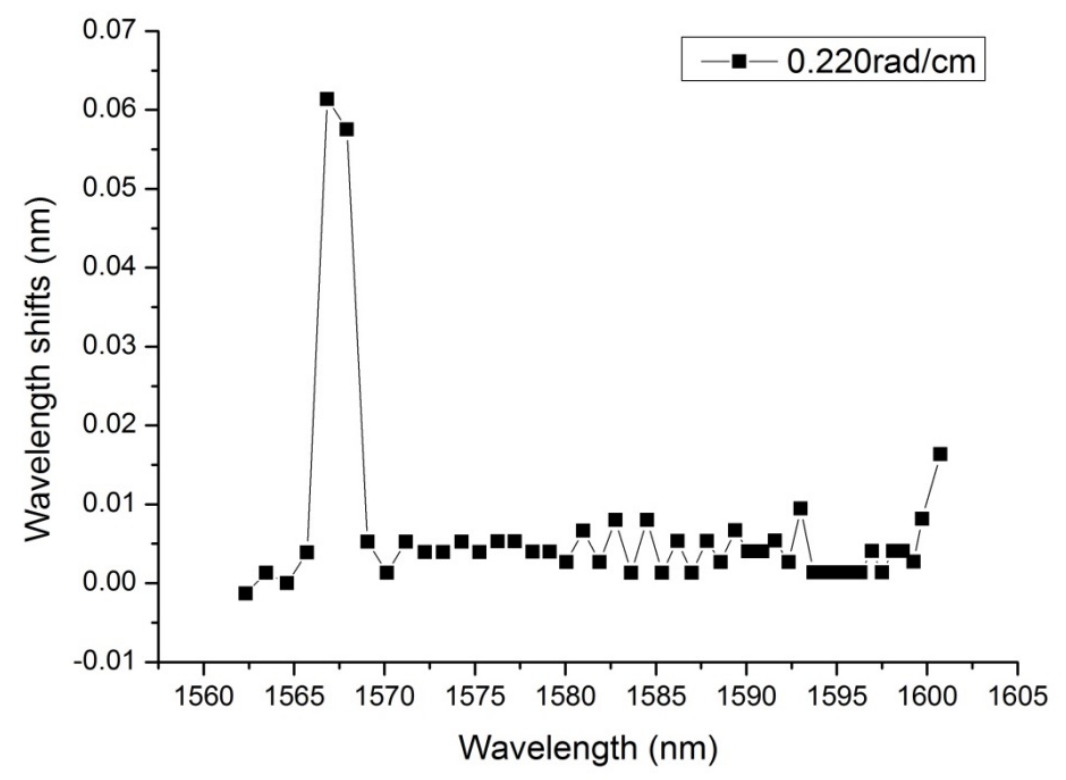

(a)Wavelength shifts VS Torsion angle

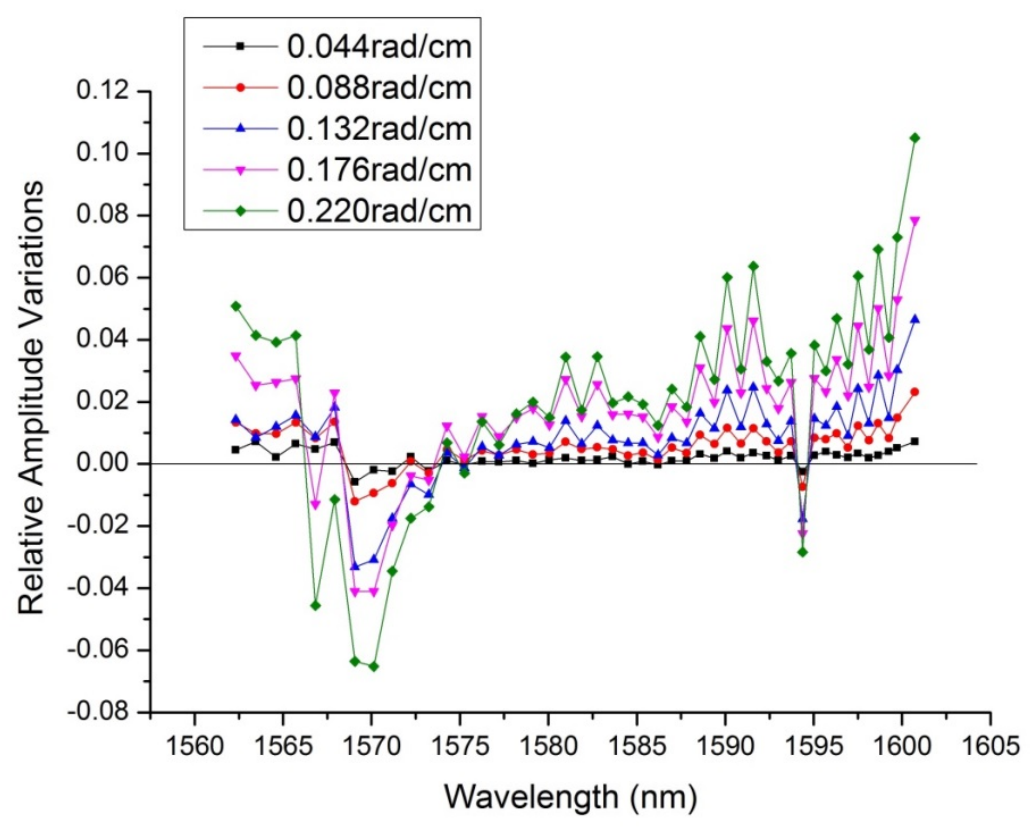

(b)Relative amplitude variations VS Torsion angle

Figure 4--6 Wavelength shifts and Relative amplitude variations

caused by $2.2 \mathrm{rad} / \mathrm{cm}$ torsion angle 
However, the amplitudes of the resonances show decreases (except several resonances at around $1570 \mathrm{~nm}$ ) while the torsion angle increases. The decreases show unordered magnitudes at numerous cladding mode resonances since each cladding mode is a complex combination of several sub modes. However, in the region between $1575 \mathrm{~nm}$ and $1593 \mathrm{~nm}$, in which the couplings are strongest, the amplitude averagely decreases $2.55 \%$ while the torsion angle reaches to $0.22 \mathrm{rad} / \mathrm{cm}$.

The increases of resonance amplitudes at around $1570 \mathrm{~nm}$ occur as a result of the deformations of resonances, which are shown in Figure 4--7. The deformations of resonances are caused by the shape distortions of grating plane structures. Two larger wavelength shifts shown in Figure 4--7(a) at around $1567 \mathrm{~nm}$ are also caused by the deformations as well. 


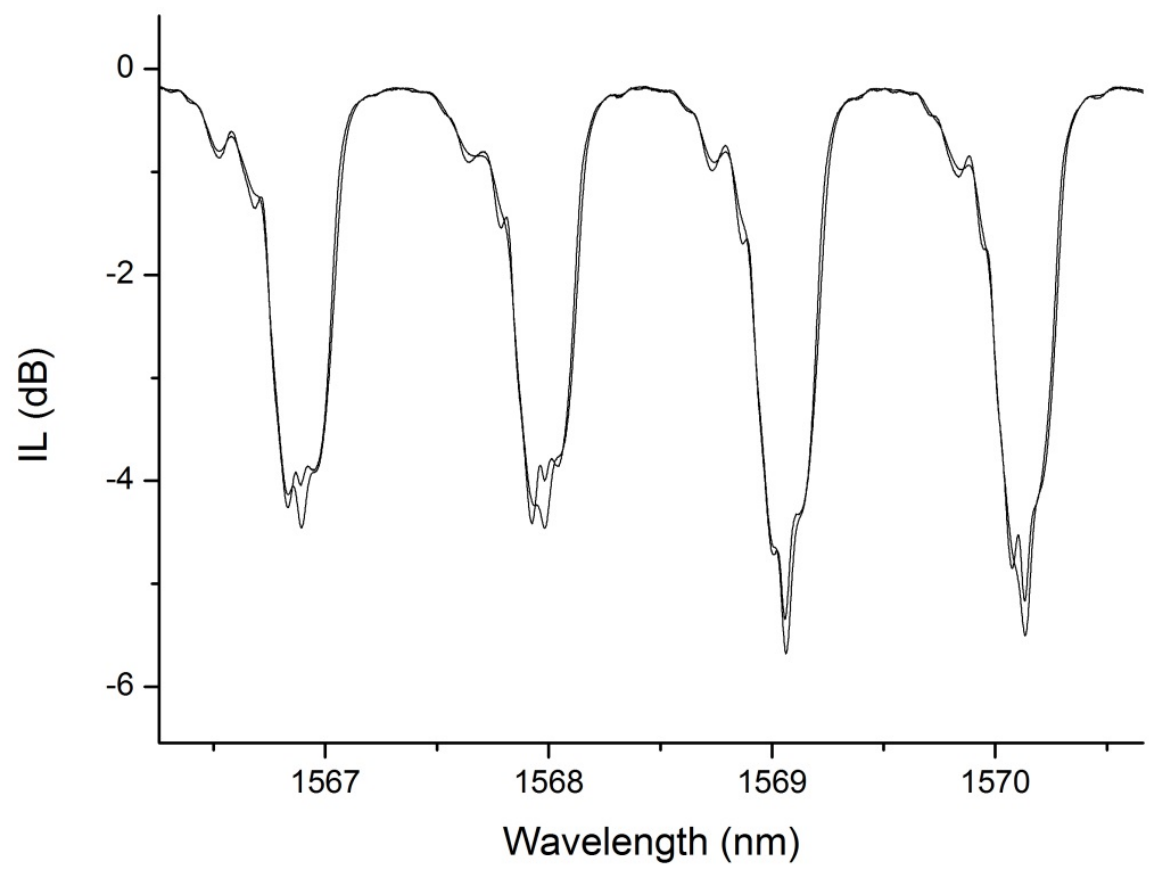

Figure 4--7 The deformations of resonances caused by torsion angle

To sum up, the increase of torsion angle applied to the TFBG does not give rise to the shifts of wavelengths of coupling resonances. However, small amplitude variations (at around $2.55 \% / 0.22 \mathrm{rad} / \mathrm{cm}$ of the amplitude) occur in a twisted TFBG as a result of the shape distortions of grating structures. Meanwhile, the Bragg mode shows strong stability as a result of its polarization-independent property. So the Bragg mode in the insertion loss spectrum is a good choice to be the reference in order to avoid the effect of strain and temperature in a twist sensor (the effects of strain do not exist since the relate wavelength shifts between Bragg mode and cladding modes are almost zero, see Chapter 3). 


\subsubsection{Polarization properties}

Compared to insertion loss spectrum, which shows non-polarization-dependent properties, PDL spectrum of TFBG shows a sensitive response to the torsion angle. Figure 4--8 shows the relative amplitude variations of the transmission PDL spectra of a $6^{\circ}$ TFBG caused by a small counter-clockwise torsion angle (from zero to $0.1 \mathrm{rad} / \mathrm{cm}$ ).

The results are oscillated. These oscillations are not noise but mode dependent coupling issues as a result of alternative $\mathrm{P}$ and $\mathrm{S}$ modes. In order to present the entire trend, Figure 4--9 shows the average relative amplitude variation versus both clockwise and counter-clockwise torsion angles.

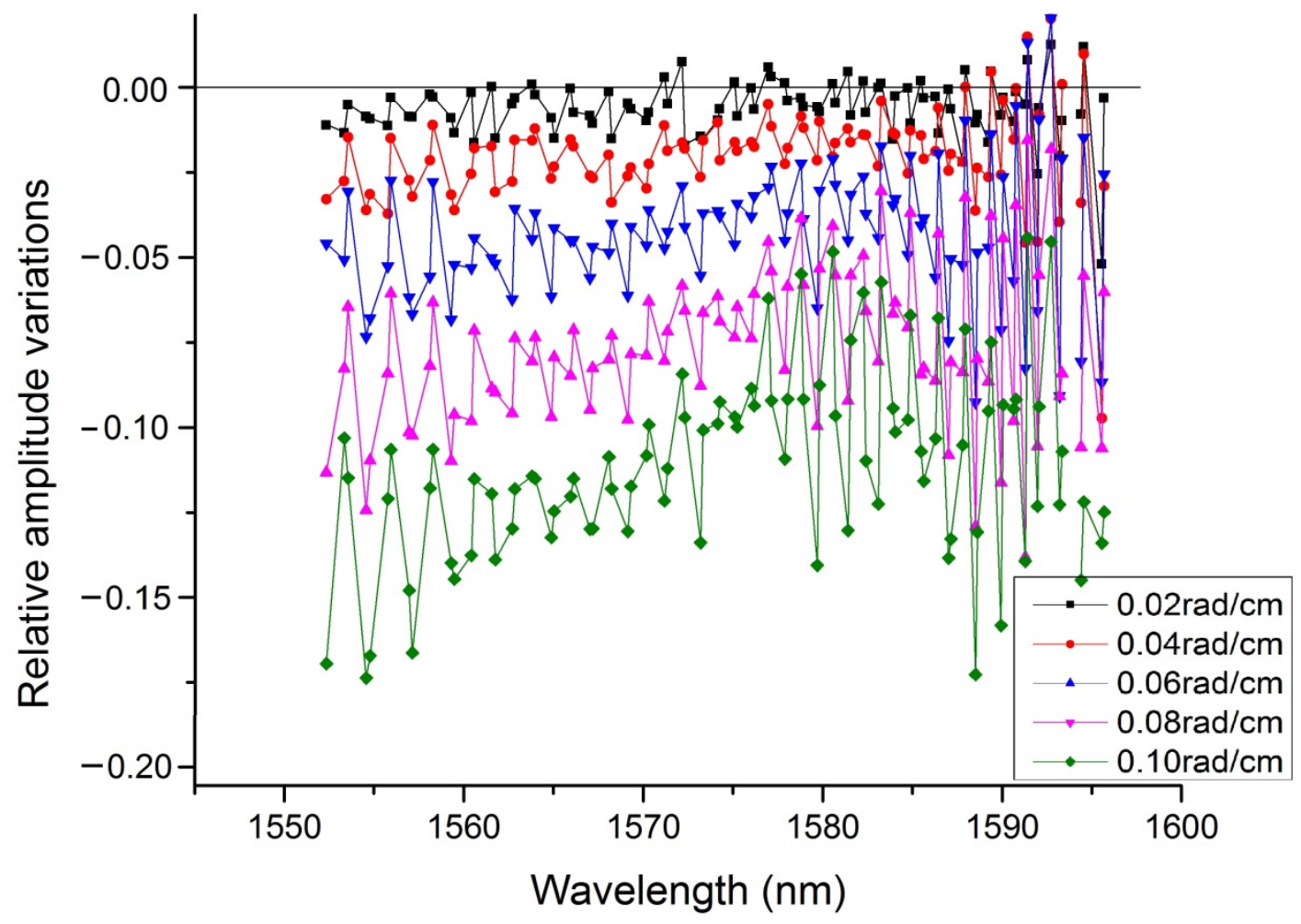

Figure 4--8 Relative amplitude variations 


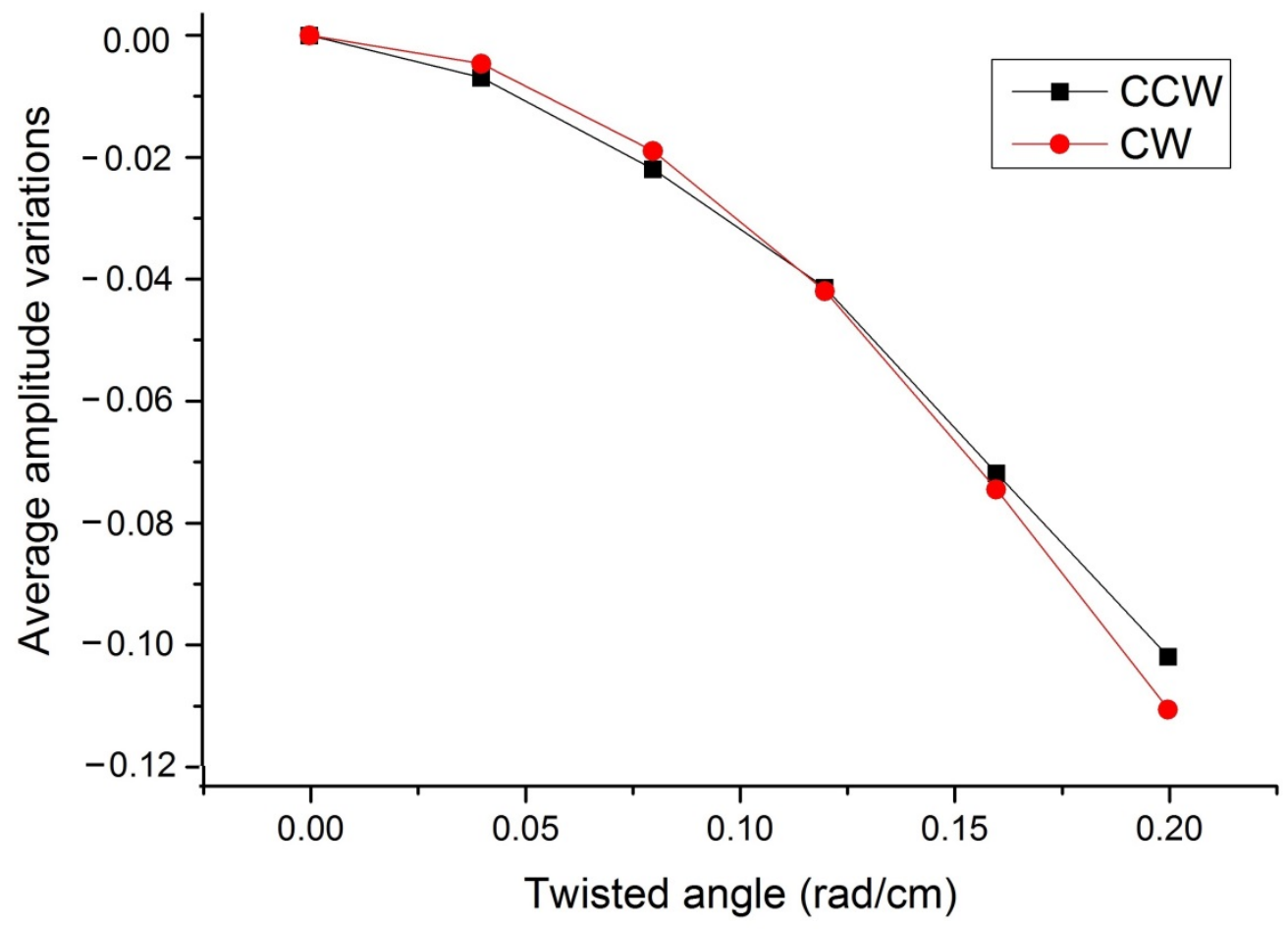

Figure 4--9 Average amplitude variations of all cladding modes between $\mathrm{CCW}$ and $\mathrm{CW}$

The average amplitude variation shows a similar trend for two rotated directions, since the decreases of amplitudes are mainly caused by the quasi-three-dimensional structure of TFBG, while the effect of birefringence is very small. In particular, TFBG breaks the fiber cylindrical symmetry structure, but provides new axial symmetric structure along the $\mathrm{x}$-axis.

The average amplitude variations of cladding modes show an accelerating increase along with the increase of torsion angle while the torsion angle is small, which is in 
accordance with the literature [45]. The average amplitude variation reaches more than $10 \%$ while the torsion angle at $0.1 \mathrm{rad} / \mathrm{cm}$ is applied to the TFBG, which is much larger than the amplitude variation of insertion loss, at $2.55 \%$ under the $0.22 \mathrm{rad} / \mathrm{cm}$ torsion angle.

\subsubsection{Accuracy analysis}

The results presented in this last section provide the oscillated amplitude variations while monitoring the numerous cladding modes. As a result, a good twist sensor could be obtained by monitoring the PDL spectrum of a single resonance, instead of the average over a large number of cladding modes. However, the PDL amplitude variation of single resonance presents tiny differences between multiple measurements. This is mainly caused by the properties of cladding modes of TFBG. First of all, polarization states are strongly dependent on the stability of the light path transmitted inside optical fiber. Any small displacement and slight touch of light path brings obvious variations of amplitude. Moreover, the entire light path cannot be straightened in experiments since the light path must form a loop from the light source to receiver. As a result, the bend of light path cannot be completely avoided and it causes changes to the polarization states. For example, the linear polarized light source may become an elliptical polarization state after a bend of light path. Then, the coupling coefficients of cladding modes are not only affected by the axial effective index modulation of TFBG, but also depend on the factor of non-axial effective index variation. It means that the details of TFBG structure cause 
the complication of cladding mode resonances which consist of various sub modes. However, both axial strain and torsion angle bring the shape change of TFBG structure and small displacement of connected fiber (clamped points, unwanted rotate and the fiber wrapped around wheels). As a result, only the overall trends of amplitude variations show monotonically increased results, while the details of cladding modes are unordered by small errors and a slight shape change of TFBG and connected fiber.

\subsection{Summary}

A twist sensor based on TFBG is introduced in this chapter. TFBG provides fiber cylindrical asymmetry structure so that the PDL amplitude variations of TFBG based on effect of torsion angle are mainly caused by the misalignments of grating planes. Obviously, the PDL amplitude variations of TFBG are much stronger than that of FBG, which are mainly caused by birefringence. As a result, TFBG is an appropriate sensitive element of twist sensor.

Compared to strain and temperature sensors, the twist sensor mainly causes the polarization-dependent amplitude decreases of cladding modes. The reduced PDL comes from the transverse misalignment of $\mathrm{P}$ and $\mathrm{S}$ orientation along the grating. The sensitivity of PDL is $10 \%$ at $0.1 \mathrm{rad} / \mathrm{cm}$. Moreover, smaller amplitude variations occur to the insertion loss, which is $2.55 \%$ at $0.22 \mathrm{rad} / \mathrm{cm}$. The variations are mainly caused by the shape distortions of grating structure.

However, PDL is sensitive to small shape distortions of TFBG as well, which cannot 
be avoided while axial strain or torsion angle is applied to the grating. This causes unordered variations of cladding mode resonances. As a result, the overall trend of amplitude variations is analyzed in this chapter. The results are linear, and follow the literature [45]. 


\section{Chapter 5 Conclusion and future work}

TFBG, as a variant of FBG, shows special characteristics such as multi-couplings, high external environment sensitivity and polarization-dependence. These characteristics mainly provided by the quasi-three-dimensional structure of TFBG, which not only reflects the forward-propagated core mode to the counter-propagated core mode, but also guides and scatters the forward-propagated core mode to numerous counter-propagated cladding modes. These characteristics bring various new functions to TFBG. As a result, the polarization-dependent strain sensor and twist sensor are described in this thesis. In particular, the advantages of the sensors based on TFBG are presented. To sum up, the advantages are:

1. Unwanted disturbances can be avoided or reduced by utilizing TFBG while sensing. The FBG sensor is a multi-purpose sensitively element so the disturbances caused by various external properties are hard to distinguish, such as the effects of strain and temperature. Normally, dual-grating structure, special fibers or special grating structures are used to solve this problem. However, the disturbances can be easily reduced by employing the relative data of TFBG because numerous modes show an independent response to external properties. In particular, the Bragg mode presents polarization-independent characteristics, which is different than other modes. So the entire shifts of spectra are 
eliminated by using the Bragg mode as a reference.

2. The polarization-dependent property brings new functions to TFBG. In particular, the property provides a TFBG sensitive response to torsion angle. Compared to insertion loss, which shows polarization-independent characteristics, the sensitivity of PDL increases one order of magnitude. Moreover, twist sensor based on TFBG shows massive amplitude variations as a result of its structure. Compared to the twist sensor based on FBG which employs the effect of birefringence, the torsion angle brings much more obvious amplitude variations to TFBG.

In closing, a problem was highlighted in this thesis. The amplitude variations, especially those in polarization-dependent spectra ( $\mathrm{P}$ and $\mathrm{S}$ modes and $\mathrm{PDL}$ ), show unordered details while considering each cladding mode separately. This is caused by the complex coupling mechanism and shape distortions of TFBG. However, the shape distortions are hard to be avoided while axial strain or torsion angle is applied to TFBG. As a result, for continuing work, the amplitude variations, mainly caused by a complex coupling mechanism, should be analyzed in order to determine the potential relationships and rules of amplitudes. 


\section{References}

[1] A. Mendez , T. F. Morse and F. Mendez; "Applications Of Embedded Optical Fiber Sensors In Reinforced Concrete Buildings And Structures." Proc. SPIE 1170, Fiber Optic Smart Structures and Skins II, 60 (February 5, 1990); doi:10.1117/12.963084.

[2] A. Kersey, "A Review of Recent Developments in Fiber Optic Sensor Technology," Optical Fiber Technology, vol. 2, no. 3, pp. 291-317, 1996.

[3] E. Joseph Friebele, "Fiber Bragg grating strain sensors: present and future applications in smart structures," Optics and Photonics News, vol. 9, no. 8, p. 33, 1998.

[4] R. Slusher, B. Eggleton and T. Strasser, "Nonlinear Pulse Reflections from Chirped fiber Grating," Optics Express, vol. 3, no. 11, pp. 465-475, 1998.

[5] V. Bhatia and A. Vengsarkar, "Optical fiber long-period grating sensors," Optics Letters, vol. 21, no. 9, pp. 692-694, 1996.

[6] C. Lin, L. Wang and G. Chern, "Corrugated long-period fiber gratings as strain, torsion, and bending sensors," Journal of Lightwave Technology, vol. 19, no. 8, pp. 1159-1168, 2001.

[7] T. Erdogan and J. Sipe, "Tilted fiber phase grating," Journal of the Optical Society of America A, vol. 13, no. 2, pp. 296-313, 1996.

[8] P. Westrook, T. Strasser and T. Erdogan, "In-line polarimeter using blazed fiber gratings," IEEE Photonics Technology Letters, vol. 12, no. 10, pp. 1352-1354, 2000.

[9] Y. Rao, T. Zhu and Q. Mo, "Highly sensitive fiber-optic torsion sensor based on an ultra-long period fiber grating," Optics Communications, vol. 266, no. 1, pp. 187-190, 2006.

[10] J. Albert, L. Shao and C. Caucheteur, "Tilted fiber Bragg grating sensors," Laser \& Photonics Reviews, vol. 7, no. 1, pp. 83-108, 2013.

[11] X. Dong, H. Zhang, B. Liu and Y. Miao, "Tilted fiber Bragg gratings: Principle and sensing applications," Photonic Sensors, vol. 1, no. 1, pp. 6-30, 2011.

[12] I. Bennion, D. Reid, C. Rowe and W. Stewart, "High-reflectivity monomode-fibre grating filters," Electronics Letters, vol. 22, no. 6, pp. 341-343, 1986.

[13] A. Vengsarkar, J. Pedrazzani, J. Judkins, P. Lemaire, N. Bergano and C. Davison, "Long-period fiber-grating-based gain equalizers," Optics Letters, vol. 21, no. 5, pp. 336-338, 1996.

[14] M. Whalen, M. Divion and R. Alferness, "Demonstration of a narrowband 
Bragg-reflection filter in a single-mode fibre directional coupler," Electronics Letters, vol. 22, no. 12, pp. 681-682, 1986.

[15] D. Lim, K. Lee, K. Kim, S. Kang, J. Ahn and M. Jeon, "Generation of multiorder Stokes and anti-Stokes lines in a Brillouin erbium-fiber laser with a Sagnac loop mirror," Optics Letters, vol. 23, no. 21, pp. 1671-1673, 1998.

[16] I. Jauncey, L. Reekie, R. Mears and D. Payne, "Narrow-linewidth fibre laser with integral fibre grating," Electronics Letters, vol. 22, no. 19, pp. 987-988, 1986.

[17] "Prof. Charles K Kao speaks on the impact of IT in Hong Kong," The Open University of Hong Kong, vol. 9, no. 1, 2000.

[18] K. Hill, Y. Fuji, D. Johnson and B. Kawasaki, "Photosensitivity in optical waveguide: Application to reflection filter fabrication," Applied Physics Letters, vol. 32, no. 10, p. 647, 1987.

[19] G. Meltz, W. Morey and W. Glenn, "Formation of Bragg grating in optical fibers by transverse holographic method," Optics Letters, vol. 14, no. 15, pp. 823-825, 1989.

[20] K. Hill, B. Malo, F. Bilodeau, D. Johnson and J. Albert, "Bragg grating fabricated in monomode photosensitive optical fiber by UV exposure through a phase mask," Applied Physics Letters, vol. 62, no. 10, pp. 1035-1037, 1993.

[21] G. MELTZ, W. MOREY, and W. GLENN, "In-fiber Bragg grating tap, " in Optical Fiber Communication, OSA Technical Digest (Optical Society of America, 1990), paper TUG1.

[22] R. Kashyap, R. Wyatt and R. Campbell, "Wideband gain flattened erbium fiber amplifier using a photosensitive fiber blazed grating," Electronics Letters, vol. 29, no. 2, pp. 154-156, 1993.

[23] C. Caucheteur, V. Voisin and J. Albert, "Polarized spectral combs probe optical fiber surface plasmons," Optics Express, vol. 21, no. 3, pp. 3055-3066, 2013.

[24] A. Othonos, "Fiber Bragg gratings," Review of Scientific Instruments, vol. 68, no. 12, pp. 4309-4341, 1997.

[25] T. Erdogan, "Fiber grating spectra," Journal of Lightwave Technology, , vol. 15, no. 8, pp. 1277-1294, 1997.

[26] K. Zhou, X. Chen, A. Simpson, L. Zhang, and I. Bennion, "High Extinction Ratio In-Fiber Polarizer Based on a 450-Tilted Fiber Bragg Grating," in Optical Fiber Communication Conference and Exposition and The National Fiber Optic Engineers Conference, Technical Digest (CD) (Optical Society of America, 2005), paper 0ME22.

[27] S. Mihailov, R. Walker and T. Stocki, "Fabrication of tilted fiber-grating polarization-dependent loss equalizer," Electronics Letters, vol. 37, pp. 284-286, 2011.

[28] M. Alam and J. Albert, "Selective excitation of radially and azimuthally polarized 
optical fiber cladding modes," Journal of Lightwave Technology, vol. 31, no. 19, pp. 3167-3175, 2013.

[29] V. Voisin, C. Caucheteur, P. Megret and J. Albert, "Interrogation technique for TFBG-SPR refractometers based on differential orthogonal light states," Applied Optics, vol. 50, no. 22, pp. 4257-4261, 2011.

[30] S. Kang, S. Kim, S. Lee, S. Kwon and S. Choi, "Temperature-independent strain sensor system using a tilted fiber bragg grating demodulator," IEEE Photonics Technology Letters, vol. 10, no. 10, pp. 1461-1463, 1998.

[31] M. Xu, J. Archambault, L. Reekie and J. Dakin, "Discrimination between strain and temperature effects using dual-wavelength fiber grating sensors," Electronics letters, vol. 30, no. 13, pp. 1085-1087, 1994.

[32] S. James, M. Dockney and R. Tatam, "Simultaneous independent temperature and strain measurement using in-fibre Bragg grating sensors," Electronics Letters, vol. 32, no. 12, pp. 1133-1134, 1996.

[33] M. Song, S. Lee, S. Choi and B. Lee, "Simulaneous measurement of temperature and strain using two fiber Bragg gratings embedded in a glass tube," Optical Fiber Technology, vol. 3, no. 2, pp. 194-196, 1997.

[34] C. Chen and J. Albert, "Strain-optic coefficients of individual cladding modes of singlemode fibre: theory and experiment," Electronics Letters, vol. 42, no. 18, pp. 1027-1028, 2006.

[35] S. Rahimi, D. Ban, G. Xiao, Z. Zhang and J. Albert, "Temperature and strain sensors based on integration of tilted fiber Bragg gratings with a free spectral range matched interrogation system," IEEE Sensors Journal, vol. 9, no. 7, pp. 858-861, 2009.

[36] C. Chen, L. Xiong, A. Jafari and J. Albert, "Differential sensitivity characteristics of tilted fiber Bragg grating sensors." Proc. SPIE 6004, Fiber Optic Sensor Technology and Applications IV, 60040B (November 10, 2005); doi:10.1117/12.629975.

[37] Y. Rao, T. Zhu and Q. Mo, "Highly sensitive fiber-optic torsion sensor based on an ultra-long period fiber grating," Optics Communications, vol. 266, no. 1, pp. 187-190, 2006.

[38] L. Shi, T. Zhu, Y. Fan, K. Chiang and Y. Rao, "Torsion sensing with a fiber ring laser incorporating a pair of rotary long-period fiber gratings," Optics Communications, vol. 284, no. 22, pp. 5299-5302, 2011.

[39] X. Chen, K. Zhou, L. Zhang and I. Bennion, "In-fiber twist sensor based on a fiber Bragg grating with 81 tilted structure," IEEE Photonics Technology Letters, vol. 18, no. 21-24, pp. 2596-2598, 2006.

[40] O. Frazao, C. Jesus, J. Baptista, J. Santos and P. Roy, "Fiber-optic interferometric torsion sensor based on a two-LP-mode operation in birefringent fiber," IEEE Photonics Technology Letters, vol. 21, no. 17, pp. 1277-1279, 2009. 
[41] H. Kim, T. Kim, B. Kim and Y. Chung, "Temperature-Insensitive Torsion Sensor With Enhanced Sensitivity by Use of a Highly Birefringent Photonic Crystal Fiber," IEEE Photonics Technology Letters, vol. 22, no. 20, pp. 1539 - 1541, 2010.

[42] W. Chen, S. Lou, L. Wang, H. Zou, W. Lu and S. Jian, "Highly Sensitive Torsion Sensor Based on Sagnac Interferometer Using Side-Leakage Photonic Crystal Fiber," IEEE Photonics Technology Letters, vol. 23, no. 21, pp. 1639 - 1641, 2011.

[43] Y. Wang, M. Wang and X. Huang, "In fiber Bragg grating twist sensor based on analysis of polarization dependent loss," Optics Express, vol. 21, no. 10, pp. 11913-11920, 2013.

[44] K. Lee and T. Erdogan, "Mode coupling in spiral fiber gratings," Electronics Letters, vol. 37, pp. 156-157, 2000.

[45] P. Reyes and P. Westbrook, "Tunable PDL of Twisted-Tilted Fiber Gratings," IEEE Photonics Technology Letters, vol. 15, no. 6, pp. 828 - 830, 2003. 